Subscriber access provided by DSM Information Services | Questions? helpdesk.infos@dsm.com

\title{
Article
}

\section{Exploring the non-conserved sequence space of synthetic expression modules in Bacillus subtilis}

Christopher Sauer, Emiel Ver Loren van Themaat, Leonie Boender, Daphne Groothuis, Rita Cruz, Leendert Willem Hamoen, Colin Harwood, and Tjeerd van Rij

ACS Synth. Biol., Just Accepted Manuscript • DOI: 10.1021/acssynbio.8b00110 • Publication Date (Web): 25 Jun 2018

Downloaded from http://pubs.acs.org on June 27, 2018

\section{Just Accepted}

"Just Accepted" manuscripts have been peer-reviewed and accepted for publication. They are posted online prior to technical editing, formatting for publication and author proofing. The American Chemical Society provides "Just Accepted" as a service to the research community to expedite the dissemination of scientific material as soon as possible after acceptance. "Just Accepted" manuscripts appear in full in PDF format accompanied by an HTML abstract. "Just Accepted" manuscripts have been fully peer reviewed, but should not be considered the official version of record. They are citable by the Digital Object Identifier (DOI®). "Just Accepted" is an optional service offered to authors. Therefore, the "Just Accepted" Web site may not include all articles that will be published in the journal. After a manuscript is technically edited and formatted, it will be removed from the "Just Accepted" Web site and published as an ASAP article. Note that technical editing may introduce minor changes to the manuscript text and/or graphics which could affect content, and all legal disclaimers and ethical guidelines that apply to the journal pertain. ACS cannot be held responsible for errors or consequences arising from the use of information contained in these "Just Accepted" manuscripts. 


\section{Exploring the non-conserved sequence space of synthetic expression}

2 modules in Bacillus subtilis

3

4 Christopher Sauer ${ }^{1,2}$, Emiel Ver Loren van Themaat ${ }^{2}$, Leonie G.M. Boender ${ }^{2}$, Daphne

5 Groothuis ${ }^{2}$, Rita Cruz ${ }^{1,2}$, Leendert W. Hamoen ${ }^{1,3}$, Colin R. Harwood ${ }^{1}$, Tjeerd van Rij ${ }^{2,4}$

6

$7{ }^{1}$ Centre for Bacterial Cell Biology, Institute for Cell and Molecular Biosciences, Newcastle

8 University Newcastle upon Tyne, United Kingdom

$9 \quad{ }^{2}$ DSM Biotechnology Center, P.O. Box 1, 2600 MA Delft, The Netherlands

$10{ }^{3}$ Swammerdam Institute for Life Sciences, University of Amsterdam, Science Park 904,

$111098 \mathrm{XH}$ Amsterdam, The Netherlands

$12{ }^{4}$ Corresponding author: Tjeerd van Rij

13

14

15

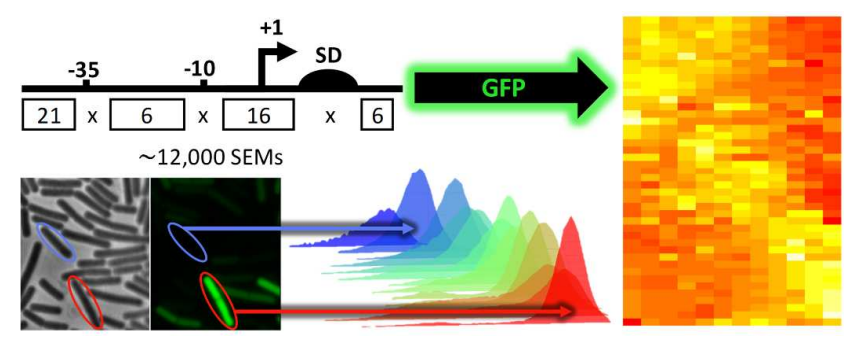

16 TOC Figure

17

18 Bacillus subtilis, FlowSeq, synthetic expression module, promoter library, GFP, xylanase 


\section{ABSTRACT}

21

22 Increasing protein expression levels is a key step in the commercial production of enzymes.

23 Predicting promoter activity and translation initiation efficiency based solely on consensus

24 sequences have so far met with mixed results. Here, we addressed this challenge using a

25 "brute-force" approach by designing and synthesizing a large combinatorial library

26 comprising $\sim 12,000$ unique synthetic expression modules (SEMs) for Bacillus subtilis. Using

27 GFP fluorescence as a reporter of gene expression, we obtained a dynamic expression

28 range that spanned five orders of magnitude, as well as a maximal 13-fold increase in 29 expression compared with that of the already strong veg expression module. Analyses of the 30 synthetic modules indicated that sequences at the 5'-end of the mRNA were the most 31 important contributing factor to the differences in expression levels, presumably by 32 preventing formation of strong secondary mRNA structures that affect translation initiation.

33 When the gfp coding region was replaced by the coding region of the $x y n A$ gene, encoding 34 the industrially relevant $B$. subtilis xylanase enzyme, only a 3-fold improvement in xylanase 35 production was observed. Moreover, the correlation between GFP and xylanase expression 36 levels was weak. This suggests that the differences in expression levels between the gfp 37 and $x y n A$ constructs were due to differences in 5'-end mRNA folding and consequential 38 differences in the rates of translation initiation. Our data show that the use of large libraries 39 of SEMs, in combination with high-throughput technologies, is a powerful approach to 40 improve the production of a specific protein, but that the outcome cannot necessarily be 41 extrapolated to other proteins. 
45 The expression of native protein encoding genes is optimized by evolutionary pressures that 46 maximize an organism's fitness in its natural environment. In contrast, industrial

47 bioprocesses aim to achieve maximum productivity of a specific target protein or enzyme in

48 a defined (artificial) environment, irrespective of the impact on fitness. For these industrial process development. However, predicting how upstream and downstream elements of a synthetic expression module will function in vivo remains a challenge. While empirical mutational studies have shown the importance of single nucleotides for modulating promoter strength and translation initiation efficiency ${ }^{1-6}$, algorithms, such as the RBS calculator, have been developed that predict translation initiation rates for diverse bacterial species including Bacillus subtilis ${ }^{7-9}$. Traditional approaches such as combining different transcriptional and translational elements, and optimizing their sequences, are time-consuming processes, and are prone to unforeseen outcomes, including heterogeneous expression across a population of cells ${ }^{10}$. Consequently, identifying expression modules that maximize transcription and translation for a protein of interest can be achieved by trial and error processes in combination with experimental design, build, test and learn cycles ${ }^{11-13}$.

The use of comprehensive synthetic DNA libraries, combined with systematic and rational engineering design principles, provides an alternative solution for current challenges in the optimization of protein expression for biotechnological purposes ${ }^{14-16}$. In this study, we employed a combinatorial design approach using a library of $\sim 12,000$ synthetic expression modules (SEMs) to improve the production of heterologous proteins in B. subtilis, a biotechnological important host. This Gram-positive bacterium is both a well-established model organism and an important industrial workhorse ${ }^{17,18}$, used for the production of various metabolites and industrial enzymes ${ }^{10,19}$.

We used the native expression module upstream of the veg gene as a basis for the construction of a synthetic expression module library containing $\sim 12,000$ unique sequences, 
71 with modifications to the upstream, downstream and intervening promoter regions. The veg 72 promoter is one of the strongest native expression modules in B. subtilis and commonly 73 used for protein expression in $B$. subtilis ${ }^{20,21}$. The resulting synthetic expression module 74 library was cloned upstream of a GFP reporter gene and analyzed by FlowSeq, a technique 75 that combines fluorescence-activated cell sorting and high-throughput DNA sequencing ${ }^{22-24}$. 76 The resulting library had a dynamic range of GFP production spanning five orders of 77 magnitude, and maximally showed a 13-fold improvement over that of the native veg 78 expression module. Following in-depth sequencing, we quantified the influence of different 79 elements of the expression module with respect to their influence on GFP expression. 80 However, when a selection of the synthetic expression modules was used to drive 81 expression of the $B$. subtilis xynA gene encoding a secretory xylanase ${ }^{25}$, the maximum 82 increase in productivity was only 3-fold. Moreover, the correlation between GFP expression 83 and XynA production was weak. Here, we discuss how sequences at the 5' end of the 84 coding sequence of both genes affects the mRNA secondary structure and the translation 85 initiation rate, and compare our measurements with the prediction of the RBS calculator ${ }^{26}$. 


\section{RESULTS \& DISCUSSION}

89 Design of a synthetic expression module (SEM) library

90 The $\sigma^{\mathrm{A}}$-dependent housekeeping $\mathrm{P}_{\text {veg }}$ promoter is one of the strongest native promoters in $B$.

91 subtilis, and not subjected to transcriptional regulation. As a consequence, the promoter is

92 active during both logarithmic growth as well as stationary growth $20,27,28$. We therefore

93 selected this promoter as the template for the design of a library of expression modules 94 (Figure 1A).

A
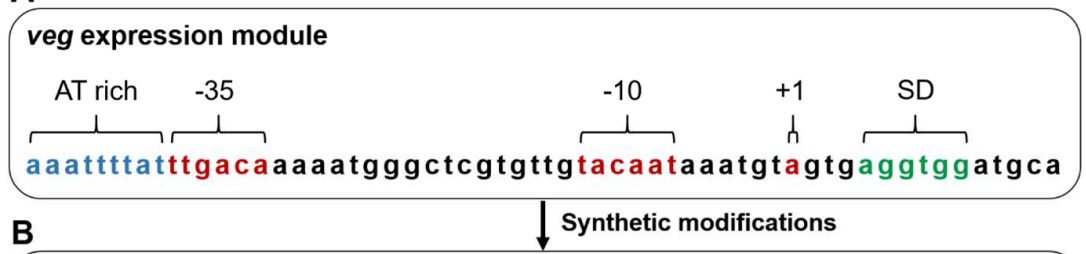

Synthetic expression module

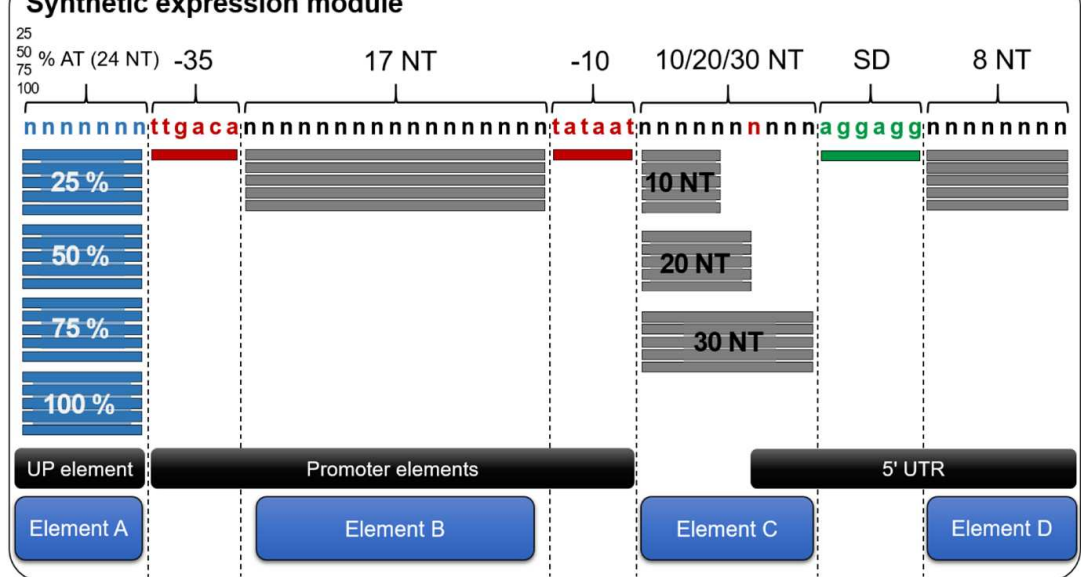

Figure 1

97 The -35 and -10 regions of $P_{v e g}$ promoter and the Shine-Dalgarno (SD) sequence of the RBS

98 are similar to the consensus for $\sigma^{A}$ recognition and pairing with $16 \mathrm{~S}$ rRNA, respectively.

99 However, the -10 region was optimized to better match the consensus sequence of $\sigma^{\mathrm{A}}$, and 100 changed from 5'-TACAAT-3' to 5'-TATAAT-3', and the SD sequence from 5'-AGGTGG-3' to 101 5'AGGAGG-3' 29. For the combinatorial design and synthesis, the expression module was 102 divided into four elements (Elements A-D, Figure 1B): Element A; the AT rich UP-element 103 located upstream of the -35 region, Element B; between the conserved "promoter elements" 
$104-35$ and -10 , Element $C$; the sequence between the -10 sequence and $S D$, and Element D;

105 the spacer between the SD and the translational start site (ATG). A higher AT content 106 upstream of the -35 region can increase the interaction between the UP-element and the $\alpha-$ 107 subunits of RNA polymerase, thereby stimulating promoter binding and transcription ${ }^{30-33}$. 108 Therefore, the 24-base pair long UP-element was randomized and twenty different 109 sequences were generated, five times four sequences with $25,50,75$ or $100 \%$ AT contents 110 (Figure 1B). For the 17-base pair spacer between the -35 and -10 regions, 5 different 111 randomized nucleotide orders were generated ${ }^{34-36}$. Translation initiation can be tuned by 112 reducing the folding of transcribed mRNAs into stable secondary structures, since strong 113 secondary structures can impede ribosome binding and therefore lower the translation 114 initiation ${ }^{37,38}$. By generating fifteen variants of the spacing between the -10 region and the 115 SD, mRNAs were obtained with different folding energies (secondary structures) at their 5'116 end. Additionally, by varying the length from 10, 20 to 30 nucleotides, the effect of local 117 distance between transcription (RNA polymerase binding) and translation initiation (ribosome 118 binding) could be analyzed. Five variants of the spacer between the SD and start codon 119 were designed to provide potential variations in translation initiation efficiency. The distance 120 of 8 nucleotides has been shown to be optimal and kept constant ${ }^{1}$. The different elements, 121 including those of the native $\mathrm{P}_{\text {veg }}$ promoter, were combined to generate a library of $\sim 12,000$ 122 synthetic expression units (Supporting Information Table S1). The library was synthesized 123 on a chip and pooled (see Methods for details).

\section{Quantification of the SEM library}

126 The library of synthetic expression modules was cloned upstream of a gfp coding region and 127 integrated as single copy at the chromosomal amyE locus (Figure 2A). Production of GFP 128 was used as a measure of expression efficiency. To monitor possible effects on the 129 metabolic load imposed by the activity of the GFP expression module, a constitutively 130 expressed mCherry reporter was used as internal reference. The mCherry expression 131 module was driven by the constitutive $\mathrm{P}_{\text {infC }}$ promoter $^{39}$ and integrated into the nprE locus of 
132 B. subtilis (Figure 2A). A library of $\sim 152,000$ clones was generated, reflecting a $\sim 13$-fold 133 clonal coverage of the $\sim 12,000$ variants in the synthetic library. The total library was grown 134 in LB to early exponential phase $\left(\mathrm{OD}_{600} \sim 0.25\right)$ and subjected to fluorescence-activated cell 135 scanning (FACScan) to determine the variations in SEM activity (Figure 2B). The GFP 136 intensities ranged over five orders of magnitude, with a profile showing a bimodal 137 distribution. In contrast, the mCherry fluorescence intensity showed a single peak indicating 138 that the protein expression capacity of individual cells was not affected at high GFP 139 expression levels.

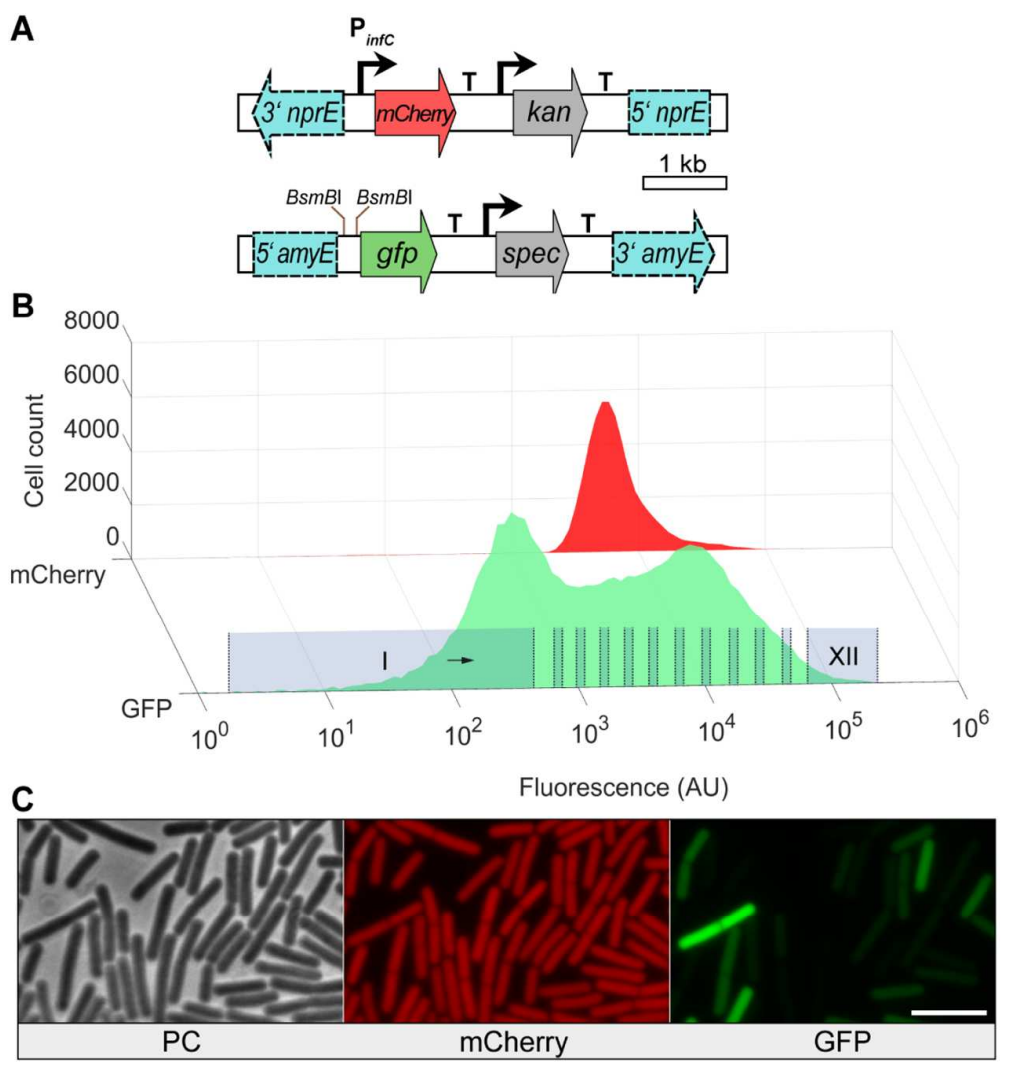

140

Figure 2

142 The wide range of GFP expression levels was confirmed by analyzing a sample containing 143 the entire library during the exponential growth phase using fluorescence microscopy (Figure 144 2C). In addition, 135 random colonies were grown overnight and their GFP over mCherry 145 fluorescence ratio was determined with a plate reader. These ratios spanned a 500-fold 146 difference in GFP expression with ratios from 10 to 0.02 (Supporting Information Figure S1). 
147 FACS was subsequently used to divide the library into 12 non-adjacent log-spaced bins of 148 increasing GFP intensities (Figure 2B, blue bins from I to XII). The population of each bin 149 was reanalyzed by FACScan (Figure $3 A-B$ ), confirming the expected GFP signal range, 150 although all bins, and especially bins $\mathrm{X}$ and $\mathrm{XI}$, showed a long tail of cells with lower GFP 151 intensities (Figure 3A). The mCherry signal was comparable to that of cells before sorting 152 (Figure 3B), but now the mCherry signal in some cells became visible in the lower intensity 153 values, possibly caused by cell differentiation ${ }^{40,41}$.
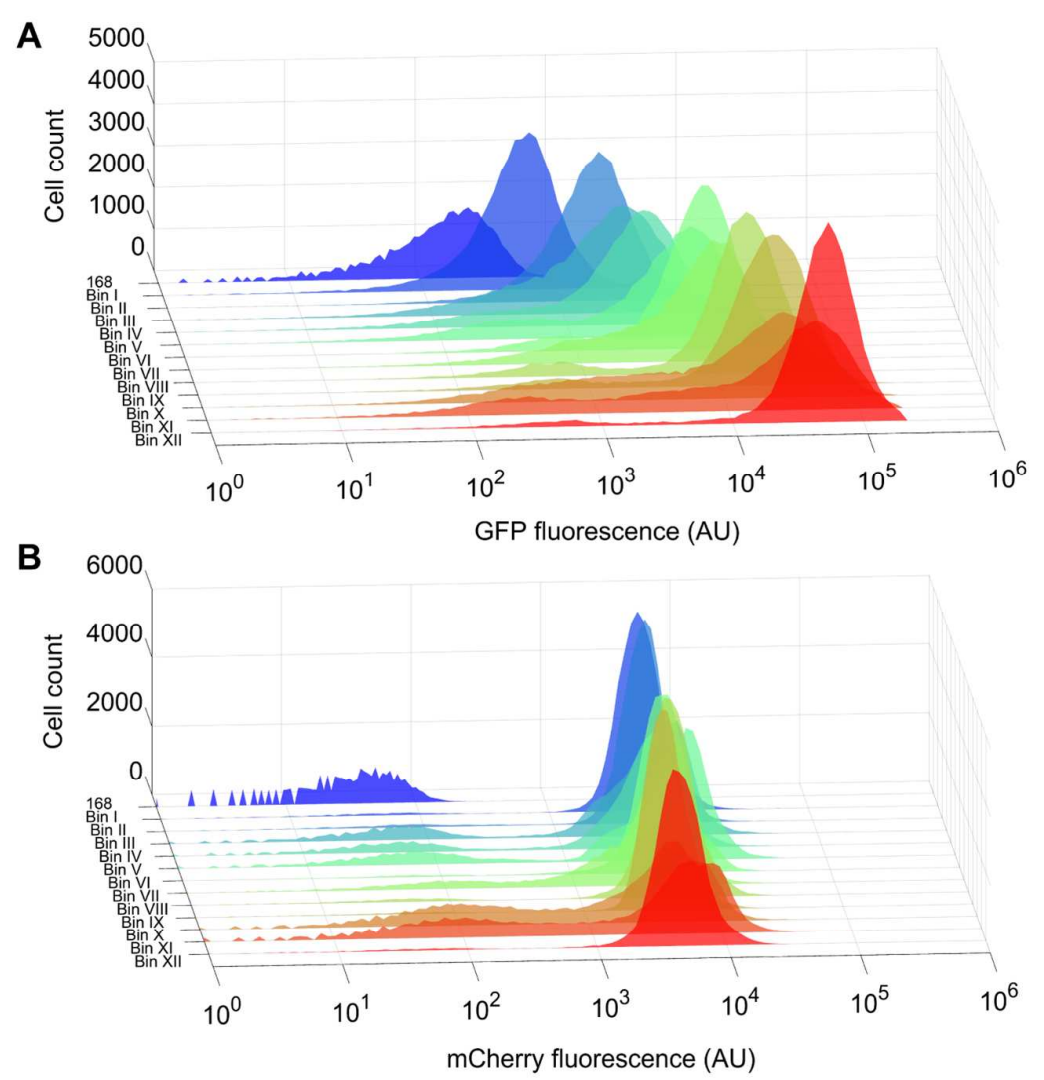

154

Figure 3

156

157 Identification and ranking of elements

158 DNA was extracted from the binned populations and SEM sequences were amplified by 159 PCR adding unique sequence barcodes for each of the twelve bins. The barcoded fragments 160 were pooled and subjected to high-throughput sequencing (Supporting Information Figure 161 S2). Of the 12,000 designed variants, $91 \%$ were identified in the sequence data. Bin I 
contained many ( 60\%) shorter sequences which represented non-functional SEM

163 sequences, presumably caused by errors during DNA synthesis. In addition, it appeared that

164 the $100 \%$ AT variants of element A were highly underrepresented, probably reflecting difficulties in synthesis, and therefore these variants were not used in further analyses. To access the sorting stringency, the distribution of nine arbitrary sequences over all bins was investigated. Indeed, each of the unique SEMs was overrepresented in only one bin (Supporting Information Figure S3). To evaluate SEMs, a score was calculated by summing the scores of each design sequence in each bin. This score was obtained by counting the presence of each SEM in a specific bin and dividing it by the total number of that SEM across all bins, multiplying the value obtained with a log10 weighted average fluorescence of each bin (Supporting Information Table S2 and Dataset S1). For instance, SEM169 has 271 sequence reads across all bins, divided over bins I-XII in the following order $4,3,2,12,7$, $116,85,21,12,1,3,5$, with most reads in bins VI and VII. The relative presence in bin I (4/271) was amplified with the log10 weighted average fluorescence of bin I (2.81). This was done for all twelve bins and these values were summed up to come to an average log10 GFP for SEM169 of 4.01. By calculating ten to the power of 4.01 the GFP expression score of 10253,82 is obtained, which falls between the average GFP fluorescence of bins VI and VII. The average GFP expression score for each SEM in the library was calculated and used for the analysis of the SEM library (Supporting Information Table S2 and Dataset S1).

\section{Contribution of individual elements}

To analyze the influence of each element on promoter strength and translation capacity, the abundance of the different elements within the twelve bins were examined. As shown in Figure 4A, the variants of Element $A$, located upstream of the -35 region (Figure 1), have only a small effect on the expression levels. It was mainly the constructs with 50 and $75 \%$ AT content (A1, A8, A12 or A13) that were overrepresented in the bins with high GFP expression levels. This suggests that the sequence upstream of the -35 region do not necessarily require an AT-rich content for an optimal interaction with the RNA polymerase. 

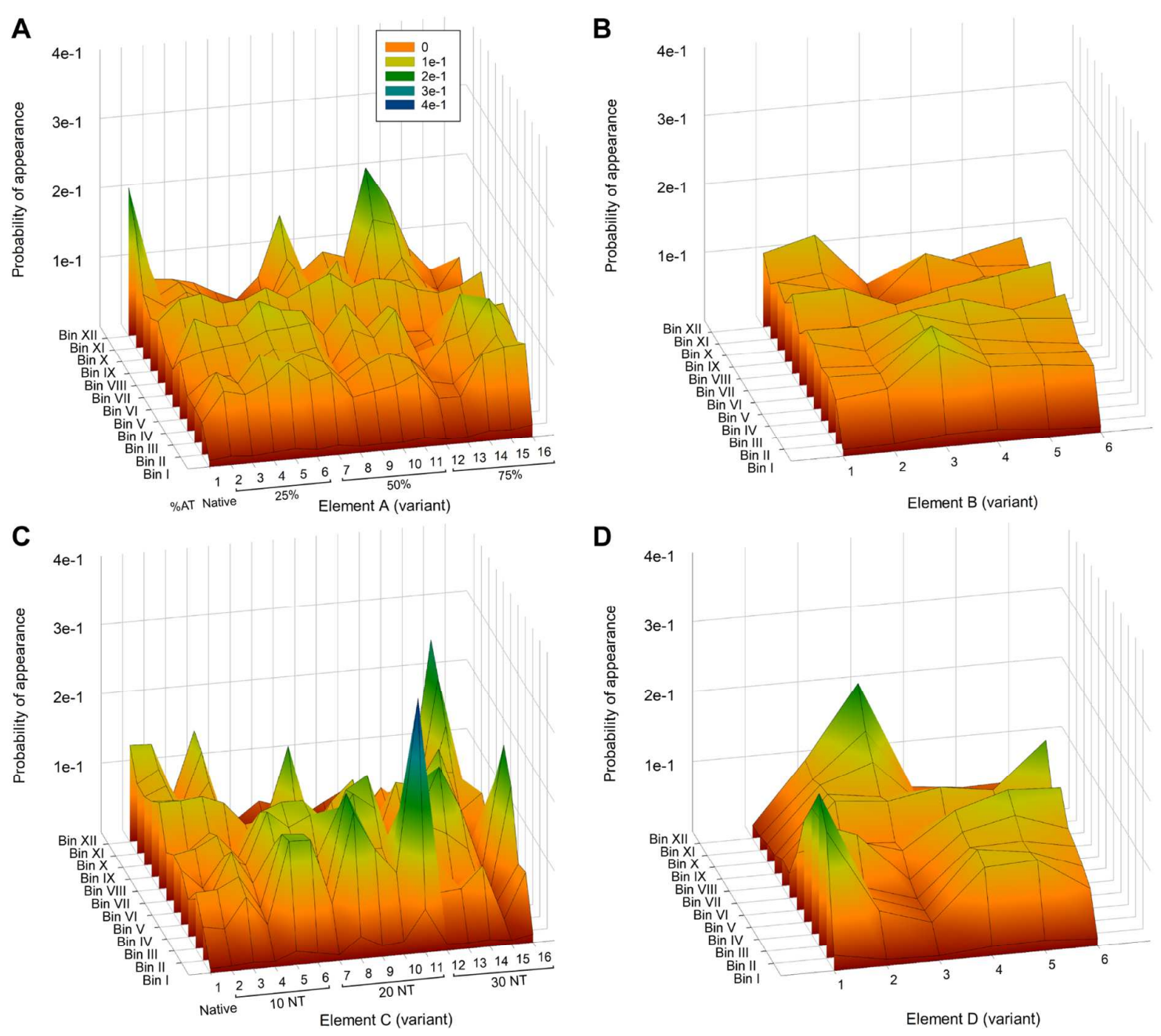

191 Figure 4

192 Analyses of Element B variants (Figure 4B), located between the -35 and -10 region (Figure

193 1), show little impact on expression levels. Nevertheless, B1 (native) and B2 are reflected in 194 bins with high GFP expression levels, while the B3 variant is found more dominantly in bins 195 with lower GFP fluorescence levels. Interestingly, B6 contains the sequence TATG from -17 196 to -14 , also called the -15 region or TGn motif, which has been shown to positively influence 197 the promoter activity in both B. subtilis and E. coli ${ }^{3,33}$. However, in our experiments this 198 element does not necessarily lead to an improved promoter activity (Figure 4B \& 5). Element 199 C variants, located between the -10 region and the SD (Figure 1), have a dominant influence 200 on GFP expression levels, with variants C1, C2, C4 and C15 being overrepresented in bins 201 VIII and higher. C15 is found in more than $20 \%$ of the cases in the higher bins. In contrast, 
202 variant C5, C6, C8, C11 and C16 are found predominantly in bins with lower GFP intensities 203 with C11 clearly overrepresented in more than $40 \%$ of the cases in bin I, reflecting a poor 204 RBS sequence. Among the Element D variants, D3 is most dominant in the high expressing 205 bins, whereas, the native sequence (element D1) is dominant in the low expressing bins. 206 The native variant of Element D, containing a 5-bp spacer, is predominant in SEMs with low 207 expression scores, confirming that spacings of 5 bp between SD and start codon is less 208 optimal for translation initiation than an 8-bp spacer ${ }^{1}$. To illustrate the distribution of element 209 variants over the twelve bins in relation to the other elements, a heatmap was created to 210 facilitate the selection of variants for low, medium or high levels of GFP production (Figure 211 5). Bin numbers are shown on the bottom with low fluorescence in Bin I and highest 212 fluorescence in Bin XII. White to yellow tiles illustrate a relative high abundance of reads and 213 red indicates low abundance or absence. The first 14 elements (A5-B4) are over 214 represented in the low expressing SEMs, the elements C11-B5 in the middle of the figure 215 are most abundant on the medium expressing SEMs while elements C2-B6 are the most 216 abundant in Bins IX-XII containing high GFP expressing SEMs. 
218 Figure 5
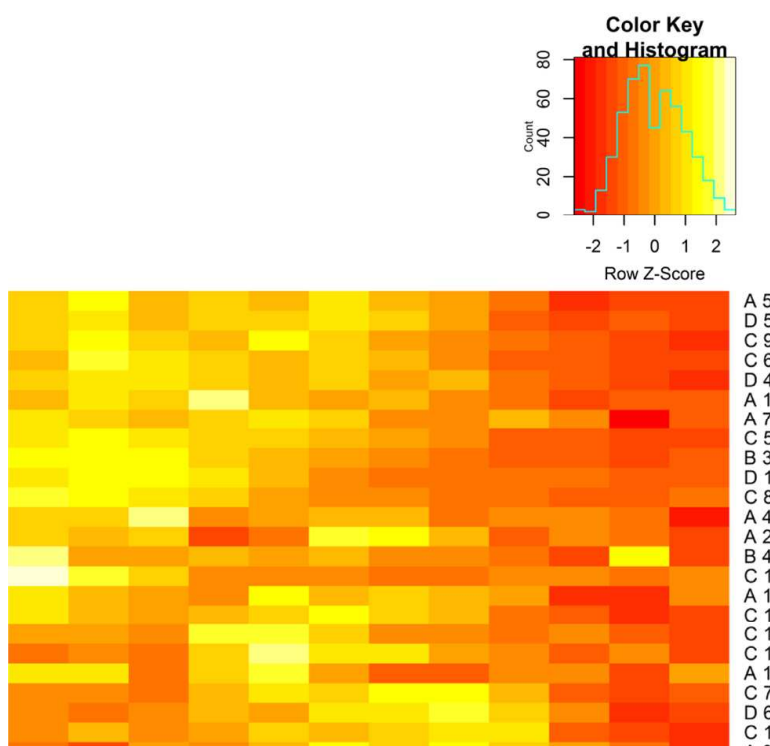

맘

-
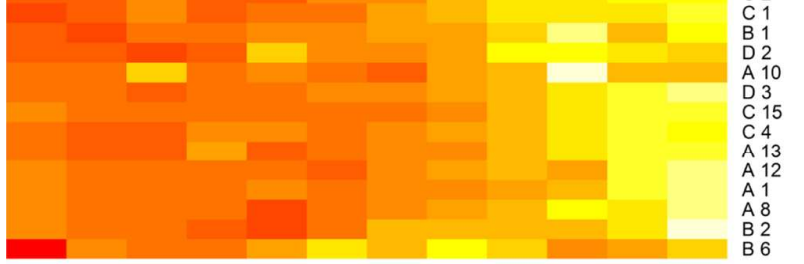

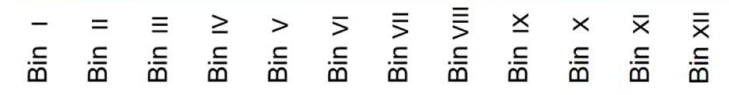

The most optimal expression system is presumably a combination of the four elements that are most abundant in bins with the highest GFP levels. To confirm this, we filtered our library in silico for SEMs that contained combinations of Element A1, A8, A12 or $\mathrm{A} 13$, Element $\mathrm{B} 1$ or $\mathrm{B} 2$, Element $\mathrm{C} 1, \mathrm{C} 2, \mathrm{C} 4$ or $\mathrm{C} 15$ and Element D3, thirty-two in total $(4 \times 2 \times 4 \times 1=32)$. These thirty-two SEMs exhibited a high level of GFP expression with an average of 4.6 (log10 scale), and a calculated score of 10.8 (Supporting Information Dataset S2). The highest GFP expression level was observed with a SEM composed of Elements A13, B2, C15, D3, which had a GFP level of expression of 4.7 (log10 scale) and a score of 11.5. This illustrates the benefits of a high-throughput combinatorial approach versus optimization of individual parts of an expression module.

It has been shown that weak secondary structures at the 5'-end of the RBS region 
230 and the first codons of the coding sequence ${ }^{42}$ correlate with expression levels ${ }^{13,24,43}$. The

231 SEM library combines sixteen $C$ elements with six $D$ elements and this resulted in 96 unique

$232 \mathrm{mRNA}$ sequences. Transcriptional starts were set at the $7^{\text {th }}$ nucleotide after the -10 promoter 233 region followed by the RBS and the first 35 nucleotides of the gfp coding region. The total 234 free energy exchange between ribosome and mRNA and translation initiation rates of these 23596 mRNA sequences were calculated with the online RBS calculator predict program ${ }^{26}$ 236 (Supporting Information Dataset S3). The measured average GFP fluorescence of each 237 mRNA sequence was plotted against the predicted translation initiation calculated with the 238 RBS calculator program (Figure 6A). Both values have a moderate positive correlation of $R$ $232^{2}=0.56$, implying some predictive value of the RBS calculator for GFP, which can still differ correlation between measured GFP and predicted translation initiation is higher or lower for certain promoter sequences. Therefore, this correlation was calculated for each of the 126 individual promoter sequences (Figure 6B, Dataset S4). The promoters containing variants B1, B2, B5 \& B6 have a similar correlation, with $R^{2}$ averages of $0.53,0.48,0.47$ and 0.53 respectively (Figure 6B, blue dots). However, the promoter containing variants B3 \& B4 249 (Figure 6B, red dots), show significantly lower $R^{2}$ values (average: B3 $\sim 0.29$, B4 0.36). For variant B3 this lower correlation can be explained by its negative effect on transcription, since SEMs with this sequence are predominantly found in Bins I-III. Increases in translation initiation and gene expression observed for $C$ and $D$ elements will not become visible in this lower range, due to the experimental set up of the bin size and distribution. For Element B4, the lack of correlation cannot be explained by a dominant negative effect on transcription since it is more evenly distributed across the bins (Figure 5). 

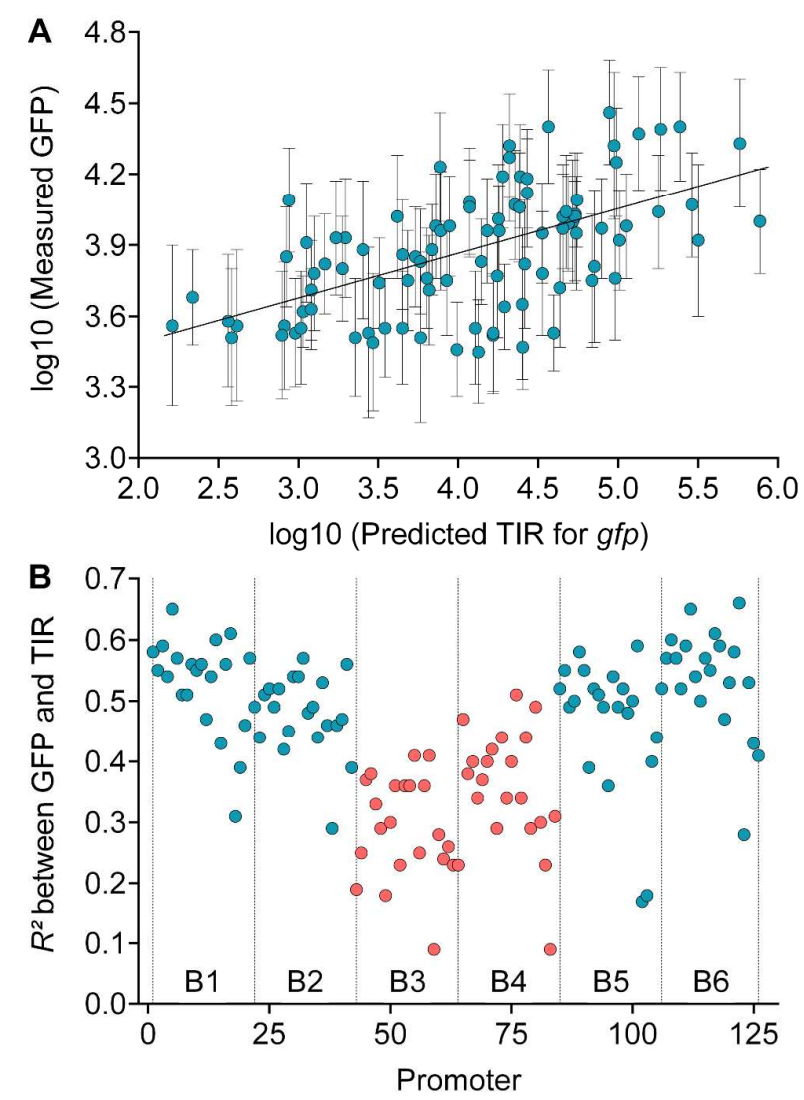

258 Figure 6

259

\section{Expression in log and stationary growth}

261 The initial FACS analysis was performed with cells grown to early exponential phase. To 262 determine whether the strongly expressing SEMs show increased GFP levels throughout the 263 exponential and stationary phases of growth, we selected 16 SEMs with high GFP expression levels on agar plates. Using a robotic $\mathrm{QPix}{ }^{\mathrm{TM}} 450$ colony picker, 16 colonies, out of a total of $\sim 2000$ colonies, were selected that displayed the highest fluorescence signal. These clones were sequenced (Supporting Information Dataset S5), and indeed they more

267 frequently included the high expression elements, C2, C4 and D3 (44\%, 31\% and 69\%, respectively).

The 16 clones were grown in a BioLector microbioreactor, monitoring biomass and fluorescence over time. A clone containing the native veg expression module was included as a reference. As shown in Figure $7 A$ and $B$, growth rates, final cell densities and mCherry 
272 levels were comparable for all 16 strains. GFP expression levels peaked towards the end of 273 exponential phase $(\sim 3 \mathrm{~h})$ with a wide range of expression levels, and decreased when the 274 stationary phase commenced (Figure 7C). Nevertheless, the differences in expression 275 between the 16 SEM variants were observed throughout the growth phases, indicating that 276 these promoter variants are not subjected to strong transcriptional regulation under these 277 growth conditions. The expression levels of the highly expressing variants 2, 9 and 16 were 278 approximately 10 to 13 -fold higher in log phase compared to the native veg expression 279 module (Figure 7C). The GFP expression values after 2, 4 and 20 hours of these 16 280 constructs, are provided in the supporting information Dataset S5.

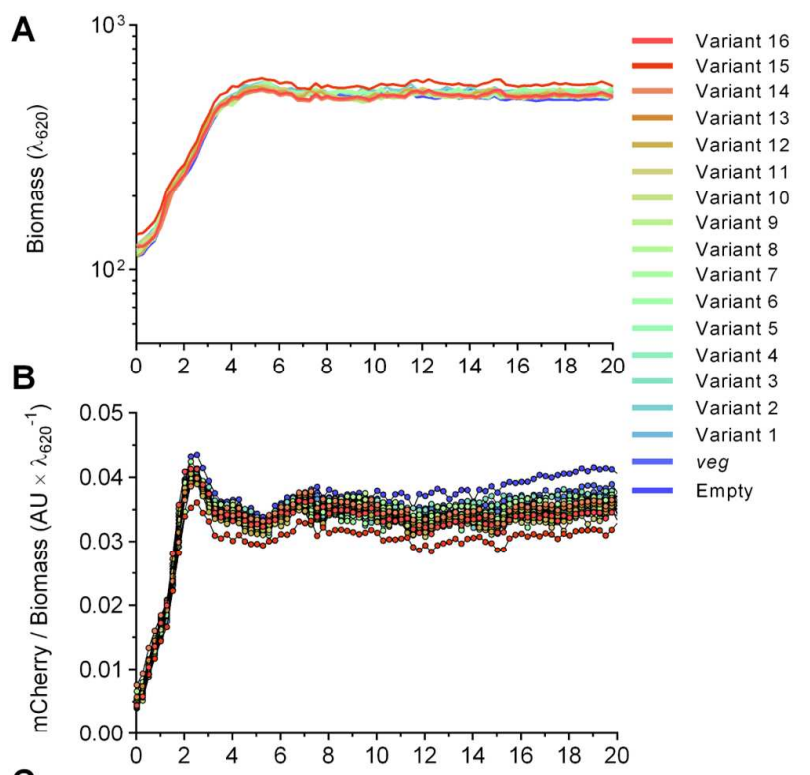

C

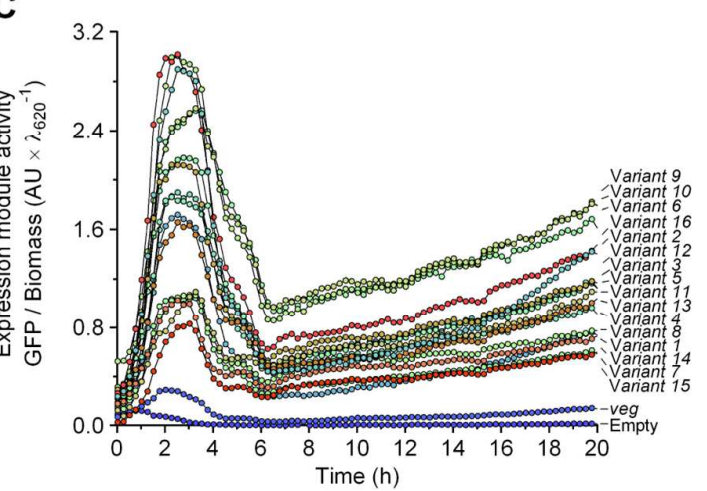

282 Figure 7 
285 To establish whether the combinatorial synthetic library approach yielded SEMs that 286 would increase production of other proteins besides GFP, we examined whether the 16 287 isolated SEM variants could increase the production of the commercially relevant secreted 288 enzyme xylanase of $B$. subtilis. This was achieved by replacing the coding sequence of the 289 gfp reporter gene downstream of the 16 SEMs with that of the commercially significant 290 xylanase gene $(x y n A)$. The enzymatic activity of secreted xylanase was quantified at three 291 time points during growth in the BioLector microbioreactor (2 h, $4 \mathrm{~h}$ and $20 \mathrm{~h})$. The XynA 292 activity at these three time points and the DNA sequences of these 16 constructs are 293 provided in the Supporting Information Dataset S5. Growth rate, biomass production and 294 mCherry expression was again similar for all strains (Supporting Information Figure S4). 295 Xylanase was produced primarily during the logarithmic phase of growth (until $4 \mathrm{~h}$ ), after 296 which the activity presumably remained constant between 4 and 20 hours. Most of the 297 selected SEMs resulted in a substantially increase in xylanase production over the native 298 veg expression module, although the highest yielding variant (variant 7 ) was only 3-fold 299 higher (Figure 8A). Furthermore, the expression level ranking of the SEM variants was 300 different for XynA and GFP. To determine the correlation between the GFP and XynA 301 production levels during logarithmic growth (2 hours), when the rate of synthesis of both 302 proteins was highest, GFP and XynA expression levels were normalized to the mCherry 303 signal and plotted against each other (Figure 8B). Only a weak correlation was observed 304 when both genes are expressed with the same SEM $\left(R^{2}=0.20\right)$. Since only the gene of 305 interest has changed, the most likely explanation for these differences is the interactions 306 between the 5' untranslated region and the coding region. This weak correlation cannot be 307 explained by post-translational events such as protein secretion or extracellular degradation 308 by proteases since these are identical for all $x y n A$ constructs ${ }^{44}$. This is supported by the 309 wide range of $\mathrm{XynA}$ activities in Figure 8A, whereas identical expression levels would be 310 expected when limitations occur in the secretion capacity or other post-transcriptional 311 processes. 

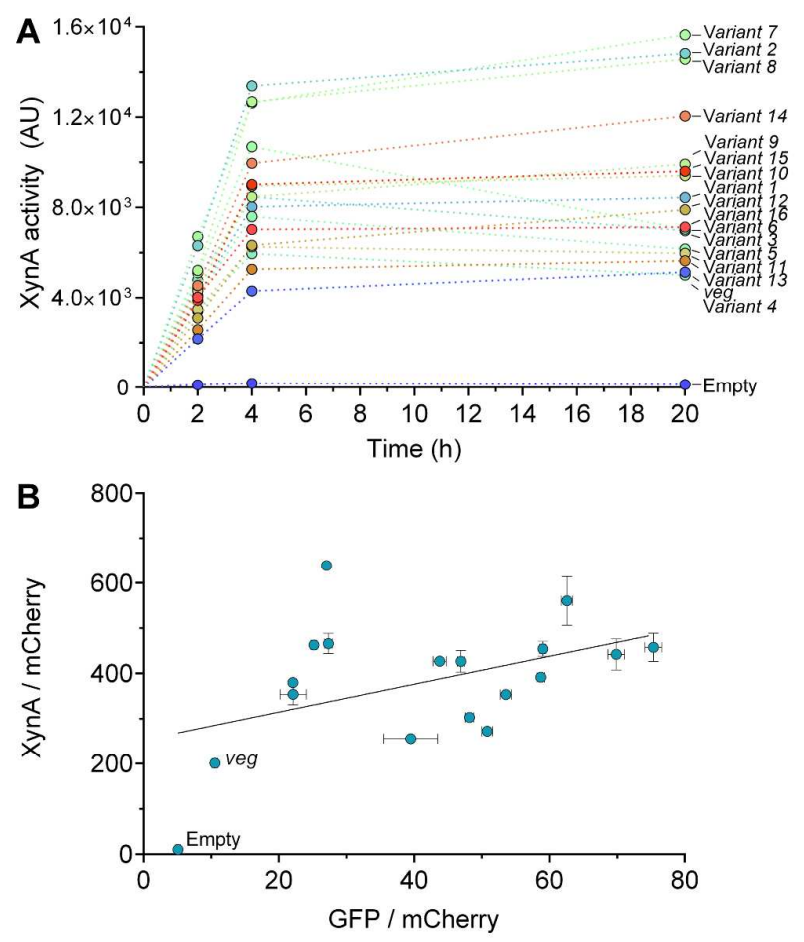

$314 \quad$ Figure 8

\section{Conclusion}

316 We have designed and used a large library of synthetic expression modules to improve 317 protein expression in B. subtilis. By combining the native veg promoter and RBS with 318 synthetic sequences modulating transcription and translation, we were able to generate GFP 319 levels with a dynamic range of over five orders of magnitudes, and a maximal 13-fold 320 increase in expression compared with the native veg sequence on which the variants were 321 based. A large part of the expression increase results from improving of the eight 322 nucleotides between the ribosome binding site and the ATG start codon. Especially for $B$. 323 subtilis, shorter SD space regions reduce the translation rate ${ }^{1}$. The extrapolation of our 324 intracellular GFP expression based data to that of a secreted protein (xylanase) yielded a 325 poor correlation. Our results confirm that optimizing and predicting gene expression levels of 326 a specific target gene cannot simply be achieved using a single reporter gene. Furthermore, 327 the coding sequence itself strongly influences the expression efficiency of the encoded 328 protein, due to the formation of secondary mRNA structures that influence, in particular, 
329 translation initiation ${ }^{6,23}$. This 5 'mRNA folding is impacted by the first codons of the coding 330 region and can affect mRNA stability and translation initiation $23,24,43$. The translation 331 initiation rate predictions with the RBS calculator includes these mRNA folding and ribosome 332 binding interactions ${ }^{26}$. Correlating the predicted and measured GFP expression levels of the 333 entire library demonstrated a moderate positive correlation with a ten-fold window of possible 334 expression levels, demonstrating that the RBS calculator can partially explain the different 335 expression levels of SEM library. Previous studies found a higher correlation $R^{2}=0.81$ for a 336 B. subtilis RBS library in combination with $\operatorname{RFP}^{7,8}$, illustrating that the predictive power of the 337 RBS calculator is context dependent, and will differ with different genes of interest. The 338 strong regulating influence of translation initiation on gene expression is supported by the 339 finding that Elements $C$ and $D$, downstream of the -10 region, had the strongest effect on 340 GFP expression. The influence of the coding region on 5' mRNA folding also explains why 341 xylanase and GFP production differed when the same expression module was used. Our 342 data illustrate that the predictive value of algorithms that predict mRNA folding and its 343 consequences for ribosome binding and translation initiation are context dependent and 344 differ greatly with each gene of interest, growth condition and sequence design method. 345 Therefore, the use of large libraries of SEMs, in combination with high-throughput screening 346 technologies, provides an effective strategy to optimize the expression and production of 347 specific proteins. 
METHODS

350

\section{Bacterial Strains and Growth Conditions}

352 For cloning purposes, NEB® 10-beta competent E. coli cells (\#C3019H, NEB®) were used.

353 Vector pCS66 was transferred to $\mathrm{dam}^{*} / \mathrm{dcm}^{-}$competent E. coli cells ( $\left.\# \mathrm{C} 2925 \mathrm{H}\right)$ to increase 354 its transformation efficiency. All analyses of the library were performed in B. subtilis strain

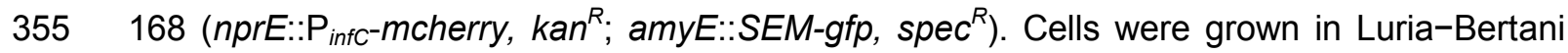
356 (LB) medium with the addition of $10 \mu \mathrm{g} / \mathrm{mL}$ kanamycin or $100 \mu \mathrm{g} / \mathrm{mL}$ spectinomycin in expression modules were cultured in a BioLector ${ }^{\circledR}$ microbioreactor ( $\mathrm{m} 2 \mathrm{p}$ labs).

361

362 overnight cultures. No antibiotics were added to the medium when cultures were assayed for expression, since the expression modules are stably integrated into the chromosome. For monitoring biomass, GFP activity and XynA production, strains containing identical

\section{Plasmid and Strain Construction}

All PCR primers are listed in the Supporting Information (Table S3). Vector pCS78, used for the integration of the $P_{\text {inf }}$-mcherry reporter at the nprE locus of 168 , was constructed in the following manner. Vector pAPNC213 ${ }^{45}$ was amplified with primers oCS001/002 to excise lacl and $\mathrm{P}_{\text {spac, }}$, resulting in pCS01. gfp was amplified from pPG49 ${ }^{46}$ with primers oCS003 and oCS004 and ligated via in pCS01 which resulted in pCS02. The kanamycin resistance cassette was amplified from pKT25N (Euromedex France SA) with primers oCS007 and oCS008 and ligated in the EcoRI and BamHI sites of pCS02, which resulted in pCS04. This vector was amplified with primers oCS009 and oCS010 and re-circularized which resulted in pCS05. The $a m p^{R}$ and ColE1 region was amplified with primers oCS027 and oCS028 and ligated, resulting in pCS08. Vector pCS08 was digested with $\mathrm{Xbal}$ and $\mathrm{BamHI}$ and the upstream flanking region of nprE was amplified with primers oCS023 and oCS024; the ligated product resulted in pCS09. The downstream flanking region of $n p r E$ was amplified with primers oCS025 and oCS026 and ligated into pCS09 digested with Notl and Sacll, this resulting in pCS10. Vector pCS10 was amplified with primers oCS045 and oCS069 to excise 
377 the gfp gene and circularized, resulting in pCS25. Vector pCS25 was amplified with primers 378 oCS048 and oCS049 to excise the Xhol site and circularized, resulting in pCS28. Vector 379 pCS28 was amplified with oCS062 and oCS071 to create pCS29. Vector pCS29 was used 380 with primers oCS077 and oCS069 to create a StarGate vector integrating in the nprE locus, 381 which resulted in pCS31. The ery ${ }^{R}$ gene of pCS31 was replaced with the $k a n^{R}$ gene resulting 382 in pCS41. The $\mathrm{P}_{\text {infC }}$-mCherry from pDOW10 ${ }^{47}$ was fused to the infC promoter and mobU SD. 383 This fragment was amplified with primers oCS523 and oCS524 and inserted in pCS41 which 384 resulted in pCS78.

Vector pCS66 was used to integrate the SEM-gfp library into the amyE locus of strain CS335. Vector pCS66 was constructed in the following manner. Vector pBest4 was amplified by PCR using primers oCS592 and oCS593 and digested with Sbfl and Notl. The chloramphenicol acetyltransferase gene flanked with $B s m B I$ restriction sites was amplified with primers oCS590 and oCS591. The amyM terminator was amplified from $B$. stearothermophilus C599 ${ }^{48}$ with the primers oCS586 and oCS587. The resulting PCR products were fused using oCS590 and oCS586 and the resulting 1-kb fragment was digested with HindIII and Notl. The spectinomycin selection marker flanked with lox66 and lox71 sites ${ }^{49}$ was amplified with primers oCS588 and oCS589 and digested with Sbfl and HindIII. Both inserts and the vector backbone were ligated, resulting in pCS75. The gfp gene was amplified from pPG49 with primers oCS594 and oCS595 and subsequently BsmBI restriction sites were introduced to allow StarGate ${ }^{\circledR}$ cloning. This resulted in vector pCS66 that was used for the insertion of SEMs to create the gfp based library and integration in the amyE locus using spectinomycin selection.

The Xylanase expression vector pCS80 was created by using Gibson Assembly ${ }^{\circledR}$ with pCS75 and the xynA gene amplified with primers oCS545 and oCS546 from the chromosomal DNA of 168.

\section{StarGate ${ }^{\circledR}$ and Library Cloning}


405 Technology Lab (Cambridge, MA 02142). The resulting oligonucleotide pool was quantified 406 using a Qubit $囚$ fluorometer with a measured concentration of $1.44 \mathrm{ng} / \mu \mathrm{l}$. The oligo pool was 407 enriched for full-length synthesis products. This step was carried out using $25 \mu \mathrm{N}$ NEBNext@ 408 high-fidelity 2× PCR master mix (cat. no. M0541S, NEB®), $19 \mu \mathrm{l}$ MilliQ water, $2.5 \mu \mathrm{l}$ of each 409 primer $(10 \mu \mathrm{M}$; oCS492/oCS493) and $1 \mu \mathrm{l}$ of the 1:100 diluted oligonucleotide pool. The 410 following PCR conditions were applied: initial denaturation at $98^{\circ} \mathrm{C}$ for $30 \mathrm{~s}, 25$ cycles 411 consisting of denaturation at $98^{\circ} \mathrm{C}$ for $10 \mathrm{~s}$, annealing at $58.4^{\circ} \mathrm{C}$ for $20 \mathrm{~s}$ and an elongation at $41272^{\circ} \mathrm{C}$ for $15 \mathrm{~s}$ followed by ta final extension at $72^{\circ} \mathrm{C}$ for $300 \mathrm{~s}$.

413 Afterwards, StarGate ${ }^{\circledR}$ cloning with $\mathrm{BsmBI}$ overhangs (IBA $\mathrm{GmbH}$ ) was performed to 414 assemble the expression vectors and the SEM library. For a simple StarGate $®$ reaction, $3 \mu \mathrm{I}$ 415 of both vector $(4 \mathrm{nM})$ and insert $(4 \mathrm{nM}-8 \mathrm{nM})$ were combined with $5 \mu \mathrm{l} 5 \times \mathrm{T} 4$ DNA ligase

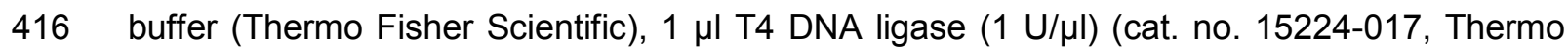
417 Fisher Scientific), $0.5 \mu \mathrm{l} E s p 3 \mathrm{I}(\mathrm{BsmBI})(10 \mathrm{U} / \mu \mathrm{l})$ (cat. no. ER0451, Thermo Fisher Scientific), 418 made up to $25 \mu \mathrm{l}$ with $12.5 \mu \mathrm{l} \mathrm{MilliQ}$ water. This mixture was incubated for $3 \mathrm{~h}$ at $30^{\circ} \mathrm{C}$, 419 another hour at $37^{\circ} \mathrm{C}$ and then used for transformation of $B$. subtilis. For the SEM library, the 420 reaction volume was doubled to $50 \mu \mathrm{l}$ and contained $12 \mathrm{nM}$ of unmethylated pCS66, $24 \mathrm{nM}$ 421 of amplified SEM DNA and $1 \mu \mathrm{l}$ Esp3I (BsmBI). $950 \mu \mathrm{l}$ of competent cells were transformed 422 using a $14 \mathrm{~mL}$ Falcon tube shaking at $37^{\circ} \mathrm{C}$ for $1 \mathrm{~h}$ before plating on $2 \times P Y$ selective agar 423 plates (BioAssay dishes, low profile, cat. no. 240845, Thermo Fisher Scientific) and 424 incubated overnight at $30^{\circ} \mathrm{C}$ wrapped in plastic foil. This cloning procedure led to the 425 creation of a library of 152,000 CFUs with $\sim 13$-fold clonal coverage. The resulting colonies 426 were scrapped off the plates, combined in a flask, and stored at $-80^{\circ} \mathrm{C}$.

\section{Xylanase assay}

429 Xylanase activities were assayed with the EnzChek® ultra xylanase assay kit (cat. no. 430 E33650, Thermo Fisher Scientific) following the suppliers protocol. The assay is based on 431 the substrate 6,8-difluoro-4-methylumbelliferyl beta-D-xylobioside, its hydrolysis of which the 432 fluorescent compound, 6,8-difluoro-4-methylumbelliferone, is released. The xylanase- 
433 containing samples were diluted 1:100 and analyzed using a plate reader (Infinite 200 Pro,

434 Tecan $®$ or FLUOstar OPTIMA, BMG LABTECH®) with excitation/emission wavelengths of $435358 / 455 \mathrm{~nm}$ after an incubation period at room temperature in the dark for 15 or $30 \mathrm{~min}$.

436

\section{Microscopy and data analysis}

438 Samples for fluorescence microscopy were grown in LB medium or on LB agar and, 439 following collection, washed immediately with PBS buffer. A sample of $0.3 \mu$ was applied to a 440 GeneFrame (AbGene, Surrey, UK) containing 1.25\% agarose in PBS. For visualization, a 441 Zeiss 200M microscope with following excitation/emission filters and timings were used: 442 GFP (470/525 nm) for $1000 \mathrm{~ms}$; mCherry $550-600 / 615-665 \mathrm{~nm}$ for $1000 \mathrm{~ms}$.

\section{Bioinformatics design}

445 For the SEM library design, sequence parts of the native $\mathrm{P}_{\text {veg }}$ were synthetically 446 modified with randomized sequence and were combined afterwards leading to a library of 44712,000 different variants (Supporting Information Dataset S6). However, specific regulatory 448 elements were conserved to ensure optimal SD and sigma factor recognition sequences. 449 The elements of the synthetic library included: (1) Element A 1-20: the UP element upstream 450 the -35 region of sigma factor recognition (TTGACA) was modified by altering the 451 percentage of AT content $(25 \%, 50 \%, 75 \%$ and $100 \%)$, (2) Element B 1-5: the spacer 452 between the -35 and -10 (TATAAT) regions was randomized with the restriction that it 453 maintained a high AT content of 75\%, (3) Element C 1-15: the spacer between position -10 454 and the SD, was randomized by using an increasing spacer length in steps of 10 from $10 \mathrm{bp}$ 455 to $30 \mathrm{bp}$ with no restriction on the \%AT content, and (4) Element D 1-5: the 8-bp-spacer 456 between SD and start codon was modified while maintaining a \%AT content of $75 \%$. The 457 associated combinatorial design, resulting in a synthetic expression module library, 458 containing 12,000 oligonucleotides, was created using the programming language Python 459 version 2.7.6. 
The experimental design was validated by generating a limited number of transformants containing SEMs. A total of 192 colonies were picked at random and sequenced to verify the library. This revealed that only $\sim 34 \%$ of the expression module sequences matched that of the bioinformatics design. The other sequences carried point mutations or deletions leading to a more diverse expression library than had previously been Information (Figure S1). The strengths of the individual expression modules were measured by determining the GFP:mCherry ratio. The data showed GFP:mCherry ratios from $\sim 10$,

472 reflecting strong expression modules, to $\sim 0.02$, revealing weak expression units. The 473 average GFP:mCherry ratio was around 1.8 and the $\sim 500$-fold difference in expression 474 strength of this limited validation set reflected an enormous dynamic expression range and a 475 large numbers of variations in the synthetic module sequences (Supporting Information 476 Figure S1), demonstrating the effectiveness of the design concept.

\section{Fluorescence-activated cell sorting (FACS)}

479 An overnight culture of the SEM library was prepared by using $5 \mathrm{~mL}$ of LB medium 480 containing the appropriate antibiotics with shaking at $165 \mathrm{rpm}$ in an Innova 2300 platform 481 shaker (New Brunswick Scientific) at $30^{\circ} \mathrm{C}$. The next day, $0.33 \mathrm{~mL}$ of overnight culture was 482 added into $100 \mathrm{~mL}$ of LB (without antibiotics) with shaking for $3 \mathrm{~h}$. After reaching an $\mathrm{OD}_{600}$ of $483 \sim 0.25$, the flask was immediately placed on ice to prevent further growth and protein folding. 484 The cells were centrifuged at $3,000 \times g$ for $10 \mathrm{~min}$ at $4^{\circ} \mathrm{C}$. The supernatant was removed 485 and the cells were resuspended in $15 \mathrm{~mL}$ of ice-cold PBS and cell chaining was reduced by 486 ultrasonication using the lowest power for $10 \mathrm{~s}$. This suspension was immediately analyzed 487 with a FACSAria ${ }^{\mathrm{TM}}$ Fusion flow cytometer system (BD Biosciences) at $4^{\circ} \mathrm{C}$ using the 
488

489

490

491

492

493

494

495

496

497

498

499

500

501

502

503

504

505

506

507

508

509

510

511

512

513

514

515

excitation/emission spectrum of 488/530-30-A nm for GFP and 561/610-20-A nm for mCherry. Additionally, the forward and side scatter parameter was applied to facilitate the measurement of cell volume and morphology, respectively, and excluding any dead our doublet cells. For the SEM library, 12 non-adjacent bins were set up with a 43-channel gap to reduce cross contaminations between the bins. The bins collected different numbers of cells as follows: bin I, one million, bins II through VIII, 250,000 cells, bins IX and X, 100,000 cells and bins $\mathrm{XI}$ and XII, 25,000 cells. After sorting, the individual bins were amplified by culturing in LB medium at room temperature overnight followed by $8 \mathrm{~h}$ at $30^{\circ} \mathrm{C}$. All bins were finally subjected to FACScan and DNA extraction for subsequent sequencing.

\section{Fluorescence-activated cell scan (FACScan)}

The FACScan technology is based on flow cytometry but only screens for the fluorescence activity of cells. For suitable FACScan analyses, cells were pelleted and subsequently resuspended in ice-cold PBS and diluted 100-fold to remove any remaining auto fluorescence of the medium. For the scanning of 50,000 events, the LSRFortessa ${ }^{\mathrm{TM}} \mathrm{X}-20$ (BD Biosciences) was applied using the excitation/emission spectrum and the forward and side scatter as done before using FACS (see above). MATLAB R2014b (MathWorks ${ }^{\circledR}$ ) was used to analyze generated data.

\section{Amplification and barcoding of the SEM library}

After sorting via flow cytometry and cultivation, chromosomal DNA was extracted from the cells in each of the 12 fluorescence intensity bins. The extracted DNA was subjected to PCR using primer oCS568 which binds on the 5'end of the amyE flanking region and primer oCS569 which binds on the first 4-11 codons of gfp, to amplify the complete expression modules and a few nucleotides of amyE and gfp. The resulting PCR products were analyzed using the Agilent DNA 1000 kit (Agilent Technologies), and amplified with forward primers oCS596 until oCS607 containing a 5 ' Illumina adapter (5'TCGTCGGCAGCGTCAGATGTGTATAAGAGACAG), bin-specific barcodes followed by the 
516 amyE generic sequence (5'-GTTCCAATACGGAGAAATCG). Primer oCS608

517 was used as reverse primer containing the Illumina adapter

518 (5'-GTCTCGTGGGCTCGGAGATGTGTATAAGAGACAG) followed by the GFP generic 519 sequence (5'-TCTTCTCCTTTGCTCATCTG).

520

\section{Sequencing of SEM library}

522 PCR products with bin-specific adapters were sequenced by Baseclear (Leiden, The 523 Netherlands) using an Illumina HiSeq 2500 as described previously ${ }^{23}$. This resulted in more 524 than 9.8 million reads of 126-bp fragments and between 151-235 Mbp of data per bin 525 (Supporting Information Figure S2). For all bins, at least $98 \%$ of the data could be matched 526 and merged into longer pseudo-reads using overlapping paired-end reads, generating 527 complete SEM sequences using FLASH version 1.2.11. FastQC version 0.11.3 (Andrews, 528 2010: www.bioinformatics.babraham.ac.uk/projects) was used for quality inspection of the 529 data and MATLAB version R2014b was used for data processing. The sequences were 530 filtered by size, with a minimum of $163 \mathrm{bp}$ matching the shortest design. The second filter 531 removed the 5'-amyE, GFP and bin adaptor sequences, reducing the sequences by an 532 additional $7 \%$. The remaining sequences were subjected to de-duplicating using MATLAB. 533 The SEM designs were converted to a FASTA file format using FASTAwrite in MATLAB. 534 This FASTA file was used in the CLC genomics workbench as reference. The design 535 sequences were trimmed to remove the generic sequences using CLC genomics workbench 536 version 8.0. Reads were mapped with 0.97 similarity over 0.9 of their length, which means 537 that $90 \%$ of the read could have a maximum of three mismatches to be mapped to the 538 reference. The mapping file (.sam) was loaded into MATLAB and for each unique read, a 539 table was generated to which a reference was mapped, i.e. several unique reads can map to 540 one library design due to the similarity constraint. An expression score for each SEM design 541 was calculated by summing the scores of each design variant in each bin and removing the 542 unique reads that are not matched to the design library. This score was calculated as 
543 follows: $\frac{n_{b i n}^{i}}{\sum_{b i n} n} x$ bin. The count $(\mathrm{n})$ of a specific design in a specific bin was divided by the

544 total count of that specific design across all bins and multiplied with the bin number.

545 Therefore, the highest score is 12 for a design only being present in bin XII. In addition, we

546 calculated the average GFP signal per SEM and for each RBS and promoter sequence.

547 First, we estimated the GFP average per bin by retrieving the GFP value at the peaks as

548 shown in the GFP histograms in Figure 3A using function "density" from the stats package in

549 R. We then calculated the GFP average per SEM using a weighted average with relative bin

550 frequencies as weights: $\frac{n_{b i n}^{i}}{\sum_{b i n} n} x \log 10\left(G F P \_b i n\right)$. Finally, we calculated the average GFP for

551 each RBS and promotor sequence by averaging all SEMs that contain the same RBS or

552 promoter. In total, we have 96 unique RBS sequences (combination of Elements $C$ and D)

553 and 126 promoter sequences (Elements $A$ and $B)$. We used $\operatorname{cor}()$ and $\operatorname{Im}()$ functions from the

554 stats package in $\mathrm{R}$ to explore the relations between the observed GFP average, promoter

555 and the predicted translation initiation rates for each RBS. Relative abundance of specific

556 designs in a bin and the average of relative abundance for each element was calculated. To

557 generate the heatmap in Figure 5, correlation was used as distance measure, hierarchical

558 clustering was performed with complete linkage as agglomeration method (function

559 heatmap.2 from library gplots) and bins were normalized by z-score.

560

561 Prediction of translation rates and total free energy exchange between ribosome and 562 mRNA

563 The RBS calculator v2.0 was used to predict translation rates of RBS sequences and total 564 free energy exchange between ribosome and mRNA using Version $2.1^{7,26}$ The mRNA 565 folding energies $(\Delta G)$ were calculated starting from the $7^{\text {th }}$ nucleotide (the transcription start 566 point) after the -10 promoter region, followed by the RBS and the first 35 nucleotides of the 567 gfp gene. The one way ANNOVA statistical analyses were performed with Minitab software 568 version 18. 


\author{
570 Supporting Information \\ 571 Table S1: Synthetic expression elements used for the SEM library. \\ 572 Table S2: Average fluorescence intensities of the twelve bins. \\ 573 Table S3: PCR primers used in this study. \\ 574 Figure S1: GFP/mCherry ratio of library variants after overnight growth. \\ 575 Figure S2: Illumina HiSeq 2500 sequence statistics of twelve bins containing SEMs. \\ 576 Figure S3: Relative abundance of nine arbitrary SEMs over the twelve bins. \\ 577 Figure S4: Growth and mCherry production of selected SEMs over time. \\ 578 Dataset S1: SEM constructs \\ 579 Dataset S2: 32 SEMs (high GFP) \\ 580 Dataset S3: RBS \\ 581 Dataset S4: Promoters \\ 582 Dataset S5: 16 SEMs fused with $x y n A / g f p$ \\ 583 Dataset S6: Oligo designs \\ 584
}




\section{ACKNOWLEDGEMENTS}

586

587 CS and RC were supported by a European Commission-funded Marie Curie Initial Training

588 Network (ITN) for Advanced Training in Industrial Enzyme Manufacturing (ATRIEM) project

589 No. 317228. We thank Marcel Hillebrand for his help and advice on the B. subtilis strain

590 construction. We thank Eric Young for his Python scripts. We thank Andrew Fuller for the

591 FACS support.

592

593 


\section{REFERENCES}

[1] Vellanoweth, R. L., and Rabinowitz, J. C. (1992) The influence of ribosome-binding-site elements on translational efficiency in Bacillus subtilis and Escherichia coli in vivo, Molecular microbiology 6, 1105-1114.

[2] Jensen, P. R., and Hammer, K. (1998) The sequence of spacers between the consensus sequences modulates the strength of prokaryotic promoters, Applied and environmental microbiology 64, 82-87.

[3] Liu, M., Tolstorukov, M., Zhurkin, V., Garges, S., and Adhya, S. (2004) A mutant spacer sequence between -35 and -10 elements makes the Plac promoter hyperactive and cAMP receptor protein-independent, Proceedings of the National Academy of Sciences of the United States of America 101, 6911-6916.

[4] Tuller, T., Carmi, A., Vestsigian, K., Navon, S., Dorfan, Y., Zaborske, J., Pan, T., Dahan, O., Furman, I., and Pilpel, Y. (2010) An evolutionarily conserved mechanism for controlling the efficiency of protein translation, Cell 141, 344-354.

[5] Davis, J. H., Rubin, A. J., and Sauer, R. T. (2011) Design, construction and characterization of a set of insulated bacterial promoters, Nucleic acids research 39 , $1131-1141$.

[6] Liebeton, K., Lengefeld, J., and Eck, J. (2014) The nucleotide composition of the spacer sequence influences the expression yield of heterologously expressed genes in Bacillus subtilis, Journal of biotechnology 191, 214-220.

[7] Espah Borujeni, A., Channarasappa, A. S., and Salis, H. M. (2014) Translation rate is controlled by coupled trade-offs between site accessibility, selective RNA unfolding and sliding at upstream standby sites, Nucleic acids research 42, 2646-2659.

[8] Farasat, I., Kushwaha, M., Collens, J., Easterbrook, M., Guido, M., and Salis, H. M. (2014) Efficient search, mapping, and optimization of multi-protein genetic systems in diverse bacteria, Molecular systems biology 10, 731.

[9] Espah Borujeni, A., and Salis, H. M. (2016) Translation Initiation is Controlled by RNA Folding Kinetics via a Ribosome Drafting Mechanism, Journal of the American Chemical Society 138, 7016-7023.

[10] Ploss, T. N., Reilman, E., Monteferrante, C. G., Denham, E. L., Piersma, S., Lingner, A., Vehmaanpera, J., Lorenz, P., and van Dijl, J. M. (2016) Homogeneity and heterogeneity in amylase production by Bacillus subtilis under different growth conditions, Microbial cell factories 15, 57.

[11] Mijakovic, I., Petranovic, D., and Jensen, P. R. (2005) Tunable promoters in systems biology, Curr Opin Biotechnol 16, 329-335.

[12] Brockmeier, U., Caspers, M., Freudl, R., Jockwer, A., Noll, T., and Eggert, T. (2006) Systematic screening of all signal peptides from Bacillus subtilis: a powerful strategy in optimizing heterologous protein secretion in Gram-positive bacteria, Journal of molecular biology 362, 393-402.

[13] Mutalik, V. K., Guimaraes, J. C., Cambray, G., Lam, C., Christoffersen, M. J., Mai, Q. A., Tran, A. B., Paull, M., Keasling, J. D., Arkin, A. P., and Endy, D. (2013) Precise and reliable gene expression via standard transcription and translation initiation elements, Nature methods 10, 354-360.

[14] Rooke, J. (2013) Synthetic biology as a source of global health innovation, Syst Synth Biol 7, 67-72.

[15] Lienert, F., Lohmueller, J. J., Garg, A., and Silver, P. A. (2014) Synthetic biology in mammalian cells: next generation research tools and therapeutics, Nat Rev Mol Cell Biol 15, 95-107.

[16] Slomovic, S., Pardee, K., and Collins, J. J. (2015) Synthetic biology devices for in vitro and in vivo diagnostics, Proceedings of the National Academy of Sciences of the United States of America 112, 14429-14435. 
[17] Harwood, C. R. (1992) Bacillus subtilis and its relatives: molecular biological and industrial workhorses, Trends Biotechnol 10, 247-256.

[18] van Dijl, J. M., and Hecker, M. (2013) Bacillus subtilis: from soil bacterium to supersecreting cell factory, Microbial cell factories 12, 3.

[19] Kulkarni, N., Shendye, A., and Rao, M. (1999) Molecular and biotechnological aspects of xylanases, FEMS microbiology reviews 23, 411-456.

[20] Radeck, J., Kraft, K., Bartels, J., Cikovic, T., Durr, F., Emenegger, J., Kelterborn, S., Sauer, C., Fritz, G., Gebhard, S., and Mascher, T. (2013) The Bacillus BioBrick Box: generation and evaluation of essential genetic building blocks for standardized work with Bacillus subtilis, Journal of biological engineering 7, 29.

[21] Guiziou, S., Sauveplane, V., Chang, H. J., Clerte, C., Declerck, N., Jules, M., and Bonnet, J. (2016) A part toolbox to tune genetic expression in Bacillus subtilis, Nucleic acids research 44, 7495-7508.

[22] Sharon, E., Kalma, Y., Sharp, A., Raveh-Sadka, T., Levo, M., Zeevi, D., Keren, L., Yakhini, Z., Weinberger, A., and Segal, E. (2012) Inferring gene regulatory logic from high-throughput measurements of thousands of systematically designed promoters, Nature biotechnology 30, 521-530.

[23] Kosuri, S., Goodman, D. B., Cambray, G., Mutalik, V. K., Gao, Y., Arkin, A. P., Endy, D., and Church, G. M. (2013) Composability of regulatory sequences controlling transcription and translation in Escherichia coli, Proceedings of the National Academy of Sciences of the United States of America 110, 14024-14029.

[24] Goodman, D. B., Church, G. M., and Kosuri, S. (2013) Causes and effects of N-terminal codon bias in bacterial genes, Science 342, 475-479.

[25] Paice, M. G., Bourbonnais, R., Desrochers, M., Jurasek, L., and Yaguchi, M. (1986) A xylanase gene from Bacillus subtilis: nucleotide sequence and comparison with $B$. pumilus gene, Archives of microbiology 144, 201-206.

[26] Salis, H. M., Mirsky, E. A., and Voigt, C. A. (2009) Automated design of synthetic ribosome binding sites to control protein expression, Nature biotechnology 27, 946950.

[27] Lam, K. H., Chow, K. C., and Wong, W. K. (1998) Construction of an efficient Bacillus subtilis system for extracellular production of heterologous proteins, Journal of biotechnology 63, 167-177.

[28] Fukushima, T., Ishikawa, S., Yamamoto, H., Ogasawara, N., and Sekiguchi, J. (2003) Transcriptional, functional and cytochemical analyses of the veg gene in Bacillus subtilis, J Biochem 133, 475-483.

[29] Osada, Y., Saito, R., and Tomita, M. (1999) Analysis of base-pairing potentials between $16 \mathrm{~S}$ rRNA and $5^{\prime}$ UTR for translation initiation in various prokaryotes, Bioinformatics $15,578-581$.

[30] Aiyar, S. E., Gourse, R. L., and Ross, W. (1998) Upstream A-tracts increase bacterial promoter activity through interactions with the RNA polymerase alpha subunit, Proceedings of the National Academy of Sciences of the United States of America $95,14652-14657$.

[31] Caramori, T., and Galizzi, A. (1998) The UP element of the promoter for the flagellin gene, hag, stimulates transcription from both SigD- and SigA-dependent promoters in Bacillus subtilis, Molecular \& general genetics : MGG 258, 385-388.

[32] Meijer, W. J., and Salas, M. (2004) Relevance of UP elements for three strong Bacillus subtilis phage phi29 promoters, Nucleic acids research 32, 1166-1176.

[33] Phan, T. T., Nguyen, H. D., and Schumann, W. (2012) Development of a strong intracellular expression system for Bacillus subtilis by optimizing promoter elements, Journal of biotechnology 157, 167-172.

[34] Moran, C. P., Jr., Lang, N., LeGrice, S. F., Lee, G., Stephens, M., Sonenshein, A. L., Pero, J., and Losick, R. (1982) Nucleotide sequences that signal the initiation of transcription and translation in Bacillus subtilis, Molecular \& general genetics : MGG 186, 339-346. 
[35] Helmann, J. D. (1995) Compilation and analysis of Bacillus subtilis sigma A-dependent promoter sequences: evidence for extended contact between RNA polymerase and upstream promoter DNA, Nucleic acids research 23, 2351-2360.

[36] Jarmer, H., Larsen, T. S., Krogh, A., Saxild, H. H., Brunak, S., and Knudsen, S. (2001) Sigma A recognition sites in the Bacillus subtilis genome, Microbiology 147, 24172424.

[37] de Smit, M. H., and van Duin, J. (1990) Secondary structure of the ribosome binding site determines translational efficiency: a quantitative analysis, Proceedings of the National Academy of Sciences of the United States of America 87, 7668-7672.

[38] $\mathrm{Na}, \mathrm{D}$., Lee, S., and Lee, D. (2010) Mathematical modeling of translation initiation for the estimation of its efficiency to computationally design mRNA sequences with desired expression levels in prokaryotes, BMC Syst Biol 4, 71.

[39] Nicolas, P., Mader, U., Dervyn, E., Rochat, T., Leduc, A., Pigeonneau, N., Bidnenko, E., Marchadier, E., Hoebeke, M., Aymerich, S., Becher, D., Bisicchia, P., Botella, E., Delumeau, O., Doherty, G., Denham, E. L., Fogg, M. J., Fromion, V., Goelzer, A., Hansen, A., Hartig, E., Harwood, C. R., Homuth, G., Jarmer, H., Jules, M., Klipp, E., Le Chat, L., Lecointe, F., Lewis, P., Liebermeister, W., March, A., Mars, R. A., Nannapaneni, P., Noone, D., Pohl, S., Rinn, B., Rugheimer, F., Sappa, P. K., Samson, F., Schaffer, M., Schwikowski, B., Steil, L., Stulke, J., Wiegert, T., Devine, K. M., Wilkinson, A. J., van Dijl, J. M., Hecker, M., Volker, U., Bessieres, P., and Noirot, P. (2012) Condition-dependent transcriptome reveals high-level regulatory architecture in Bacillus subtilis, Science 335, 1103-1106.

[40] Veening, J. W., Smits, W. K., and Kuipers, O. P. (2008) Bistability, epigenetics, and bethedging in bacteria, Annual review of microbiology 62, 193-210.

[41] Grimbergen, A. J., Siebring, J., Solopova, A., and Kuipers, O. P. (2015) Microbial bethedging: the power of being different, Current opinion in microbiology 25, 67-72.

[42] Bentele, K., Saffert, P., Rauscher, R., Ignatova, Z., and Bluthgen, N. (2013) Efficient translation initiation dictates codon usage at gene start, Molecular systems biology 9 , 675.

[43] Plotkin, J. B., and Kudla, G. (2011) Synonymous but not the same: the causes and consequences of codon bias, Nat Rev Genet 12, 32-42.

[44] Westers, H., Westers, L., Darmon, E., van Dijl, J. M., Quax, W. J., and Zanen, G. (2006) The CssRS two-component regulatory system controls a general secretion stress response in Bacillus subtilis, The FEBS journal 273, 3816-3827.

[45] Morimoto, T., Loh, P. C., Hirai, T., Asai, K., Kobayashi, K., Moriya, S., and Ogasawara, N. (2002) Six GTP-binding proteins of the Era/Obg family are essential for cell growth in Bacillus subtilis, Microbiology 148, 3539-3552.

[46] Gamba, P., Jonker, M. J., and Hamoen, L. W. (2015) A Novel Feedback Loop That Controls Bimodal Expression of Genetic Competence, PLoS genetics 11, e1005047.

[47] Detert Oude Weme, R. G., Kovacs, A. T., de Jong, S. J., Veening, J. W., Siebring, J., and Kuipers, O. P. (2015) Single cell FRET analysis for the identification of optimal FRET-pairs in Bacillus subtilis using a prototype MEM-FLIM system, PloS one 10, e0123239.

[48] Diderichsen, B., and Christiansen, L. (1988) Cloning of a maltogenic alpha-amylase from Bacillus stearothermophilus, FEMS microbiology letters 56, 53-60.

[49] Yan, X., Yu, H. J., Hong, Q., and Li, S. P. (2008) Cre/lox system and PCR-based genome engineering in Bacillus subtilis, Applied and environmental microbiology 74 , $5556-5562$. 


\section{FIGURE LEGENDS}

751

752 Figure 1: Design of Synthetic Expression Module (SEM) library. (A) Sequence of the 753 veg native expression module. (B) SEM design with four variable elements: Element A (UP754 element), Element B (spacer between -35 and -10 region [red]), Element $C$ (spacer between $755-10$ region and SD) and Element $D$ (spacer between SD and start codon). The elements 756 were modified as follows: Element A reflecting the AT content (blue) of $25 \%, 50 \%, 75 \%$, $757100 \%$ (5 variants each), Element B (5 variants), Element C (15 variants) including the +1 758 region (red), and Element D, the 8 NT spacer (5 variants). Nucleobases ( $n$ ) are reflected by 759 adenine, thymine, guanine or cytosine.

760

761 Figure 2: Fluorescence analysis of the SEM library. (A) The mcherry gene under the 762 control of the infC promoter $\left(\mathrm{P}_{\text {infC }}\right)$ and the kanamycin selection marker $(k a n)$ were integrated 763 in the nprE locus. The gfp gene under control of the SEM library and the spectinomycin $764(s p e c)$ selection marker were integrated in the amyE locus. Terminators $(T)$ prevent read765 through into adjacent genes. BsmBI restriction sites indicate the insert location of the SEMs 766 upstream of gfp. Disrupted integration loci (amyE; nprE) are indicated with dashed lines. (B)

767 GFP and mCherry fluorescence levels of the SEM library. The expression library of 152,000 768 clones was grown until early exponential phase. mCherry fluorescence levels are shown in 769 red and GFP fluorescence levels in green. Cells were sorted into 12 non-adjacent log770 spaced bins (I to XII) with increasing GFP intensities indicated by blue shades (top). (C) 771 Microscopic analysis of the unsorted SEM library during exponential growth. Showing from 772 left to right: phase contrast (PC), homogeneous red fluorescence (mCherry) and 773 heterogeneous green fluorescence (GFP). Scale bar is $5 \mu \mathrm{m}$.

774

775 Figure 3: GFP and mCherry fluorescence of bins I to XII. The populations of the 12 bins 776 and the parent strain 168 were cultured and 50,000 events of each population were 777 analyzed by FACScan. (A) The cell counts of GFP fluorescence and (B) mCherry 
778 fluorescence intensities are shown.

780 Figure 4: Abundance of SEM Elements in bins I to XII. The abundance of Element A,

781 Element $B$, Element $C$ and Element $D$ variants over the twelve bins were quantified and

782 normalized against their total counts in the SEM library, respectively. Bin I represents the 783 lowest GFP fluorescence and bin XII the highest GFP fluorescence. The frequencies of (A)

78416 Element A variants with different \%AT content, (B) 6 Element B variants, (C) 16 Element

$785 \mathrm{C}$ variants with different spacer length and (D) 6 Element $\mathrm{D}$ variants are shown. Variant 1 786 represents the native veg expression module. The probabilities of appearance are color 787 coded and shown in panel A.

788

Figure 5: Heat map of all element variants distributed over the 12 bins. Hierarchical clustering of the elements using correlation as distance metric and complete linkage as 791 agglomeration method. Bins are normalized by Z-scores. High relative abundance is white 792 and low relative abundance is red.

793

Figure 6: Measured GFP levels and predicted translation initiation. (A) The log10 of the average GFP expression level of the 96 RBS modules (sixteen C elements times six D elements $=96$ ) including their standard deviation was plotted against the log10 of the predicted translation initiation rate (TIR) calculated by the RBS calculator V2.0 ${ }^{26}$ and resulted in a moderate positive correlation $\left(R^{2}=0.56\right)$. (B) Correlating GFP expression and predicted translation initiation rate (TIR) for each of the 126 promoter sequences on the $\mathrm{x}$-axes (twenty-one A elements times six B elements $=126$ ). First 32 promoters with Elements B1 and B2 are colored blue, promoters 33-64 with Elements B3 and B4 are colored red and promoters 65-96 with Elements B5 and B6 are colored blue.

803

804 Figure 7: Growth, mCherry and GFP over time. Strains containing sixteen selected SEMs 805 and control strains carrying no expression unit (empty) or native expression module (veg) 
806 were analyzed during growth. (A) Biomass increase, (B) production of mCherry over 807 biomass and (C) SEM activity reflected by GFP fluorescence over biomass are shown. 808 Strains were grown in quadruplicate in a BioLector ${ }^{\circledR}$ microbioreactor for $20 \mathrm{~h}$ in LB medium 809 and the average values are shown.

810

811 Figure 8: Xylanase production of selected SEMs and analysis of interaction between

812 GFP and XynA expression. Strains containing 16 expression modules and control strains 813 carrying no expression module (empty) or the native expression module (veg) were analyzed 814 for (A) XynA activity in the culture medium during exponential (2 hours), transition (4 hours) 815 and stationary phase (20 hours). (B) Correlation analysis between XynA and GFP production 816 levels with respect to mCherry expression during logarithmic growth phase (2 hours) $\left(R^{2}=\right.$ 817 0.20). Error bars indicate the standard deviation of four biological replicates.

818 


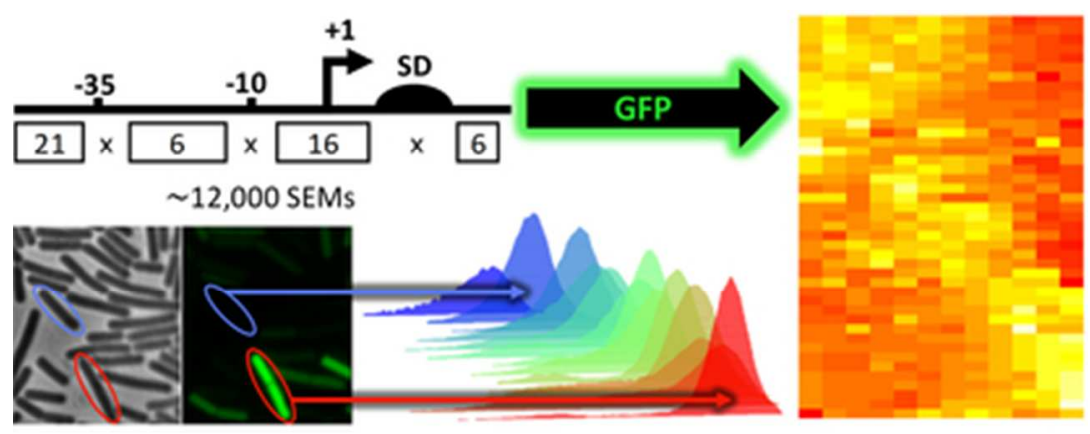

$34 \times 13 \mathrm{~mm}(300 \times 300$ DPI $)$ 

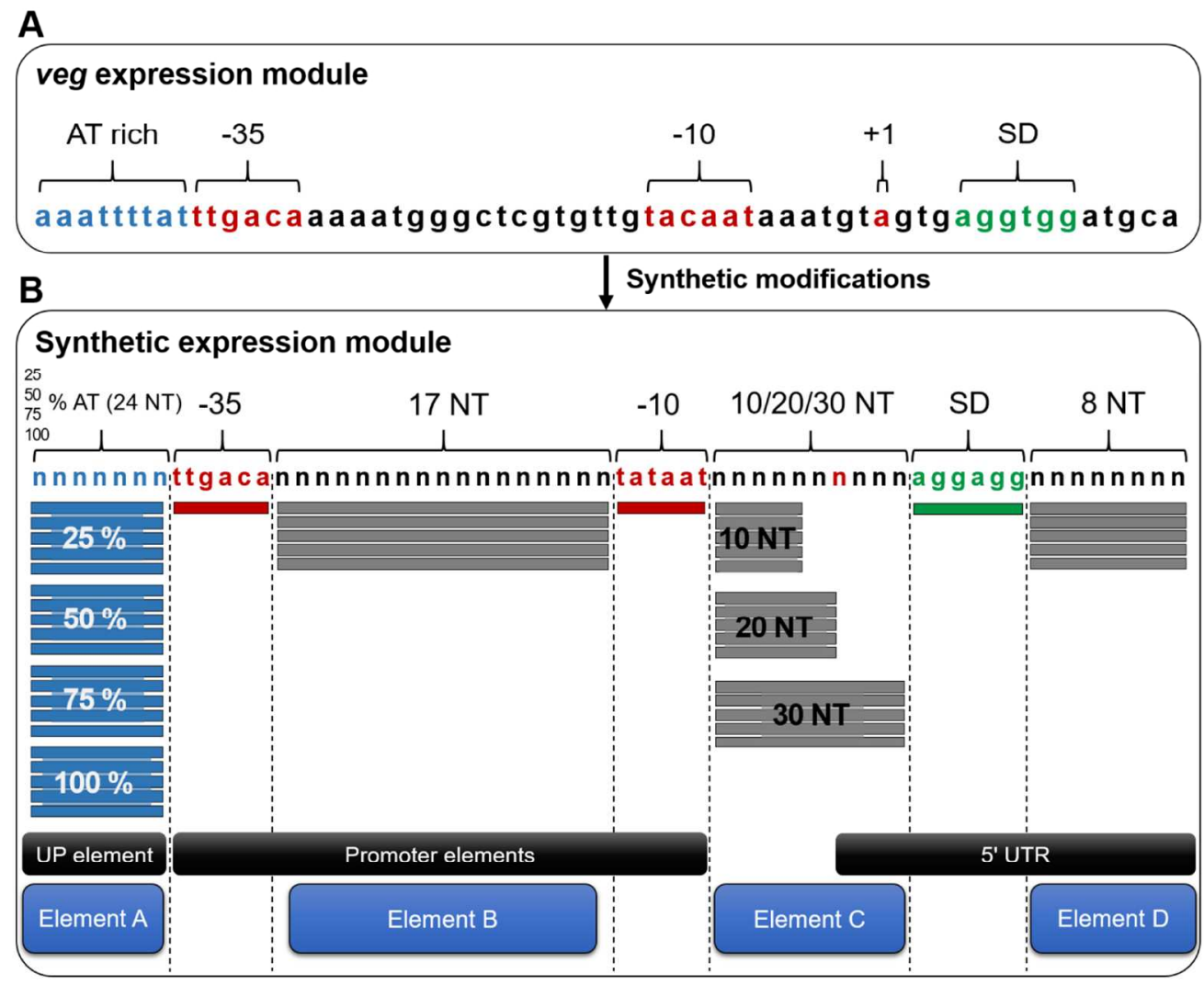

Figure 1: Design of Synthetic Expression Module (SEM) library. (A) Sequence of the veg native expression module. (B) SEM design with four variable elements: Element A (UP-element), Element B (spacer between 35 and -10 region [red]), Element $C$ (spacer between -10 region and SD) and Element $D$ (spacer between $\mathrm{SD}$ and start codon). The elements were modified as follows: Element A reflecting the AT content (blue) of $25 \%, 50 \%, 75 \%, 100 \%$ ( 5 variants each), Element B (5 variants), Element C (15 variants) including the +1 region (red), and Element D, the 8 NT spacer ( 5 variants). Nucleobases ( $\mathrm{n}$ ) are reflected by adenine, thymine, guanine or cytosine.

$116 \times 95 \mathrm{~mm}(300 \times 300$ DPI $)$ 
A
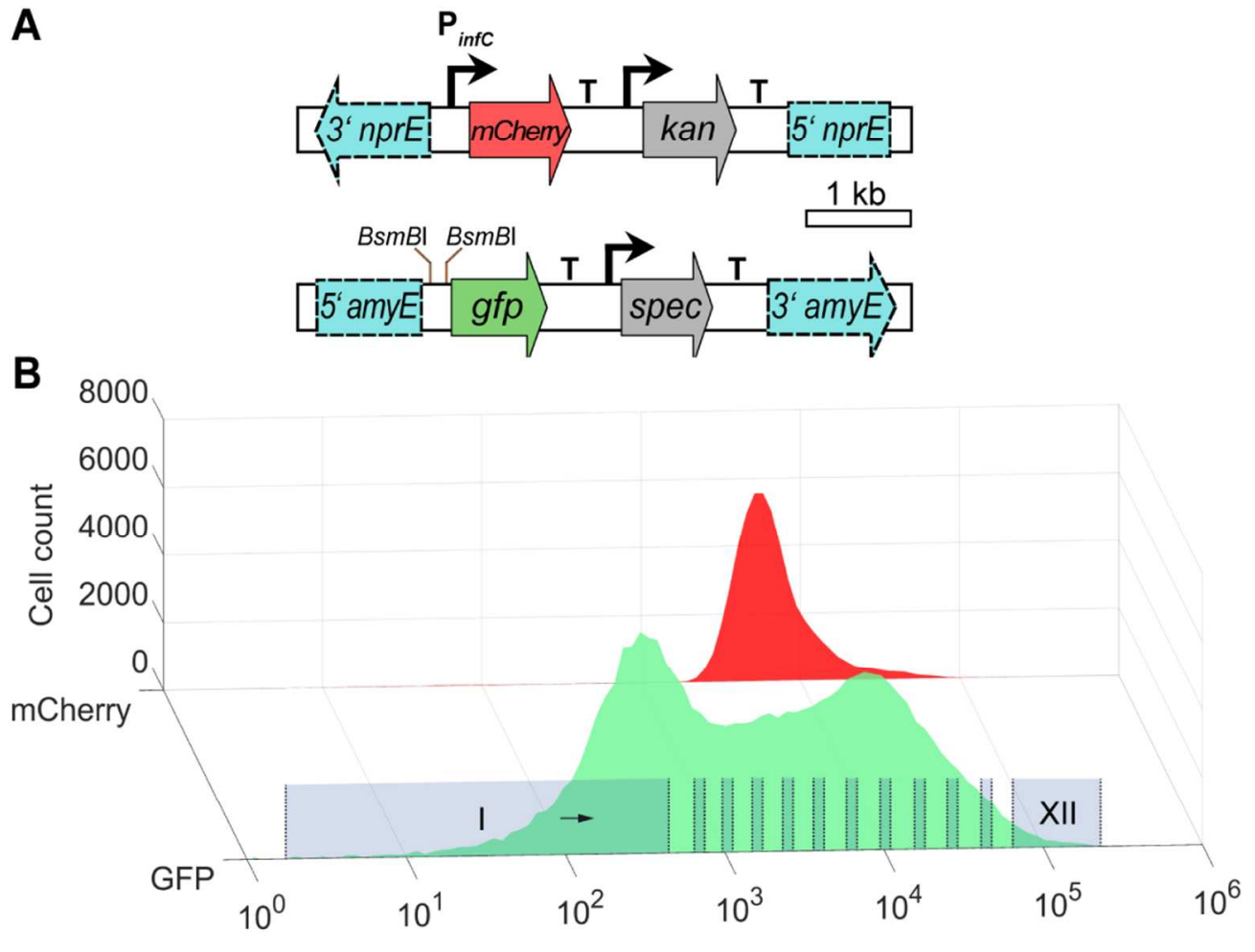

C

Fluorescence (AU)

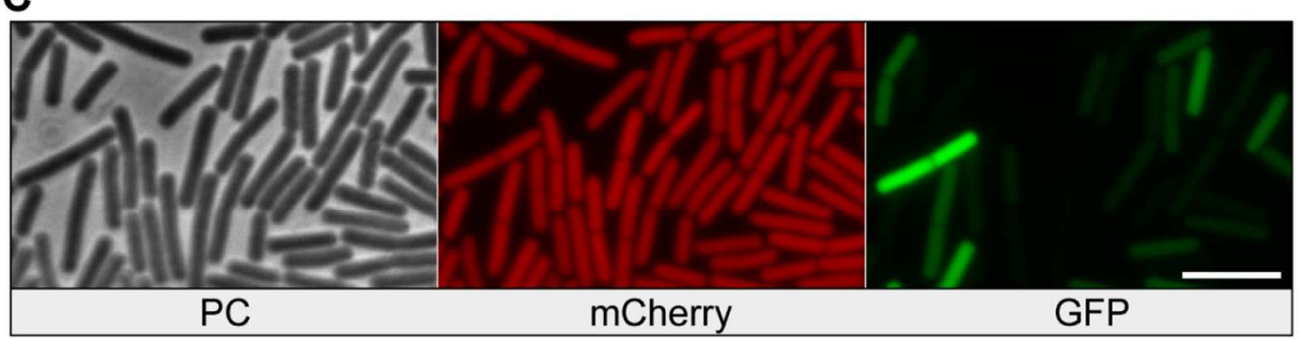

Figure 2: Fluorescence analysis of the SEM library. (A) The mcherry gene under the control of the infC promoter (PinfC) and the kanamycin selection marker (kan) were integrated in the nprE locus. The gfp gene under control of the SEM library and the spectinomycin (spec) selection marker were integrated in the amyE locus. Terminators $(T)$ prevent read-through into adjacent genes. BsmBI restriction sites indicate the insert location of the SEMs upstream of gfp. Disrupted integration loci (amyE; nprE) are indicated with dashed lines. (B) GFP and mCherry fluorescence levels of the SEM library. The expression library of 152,000 clones was grown until early exponential phase. mCherry fluorescence levels are shown in red and GFP fluorescence levels in green. Cells were sorted into 12 non-adjacent log-spaced bins (I to XII) with increasing GFP intensities indicated by blue shades (top). (C) Microscopic analysis of the unsorted SEM library during exponential growth. Showing from left to right: phase contrast (PC), homogeneous red fluorescence (mCherry) and heterogeneous green fluorescence (GFP). Scale bar is $5 \mu \mathrm{m}$.

$107 \times 113 \mathrm{~mm}(300 \times 300 \mathrm{DPI})$ 
A 5000

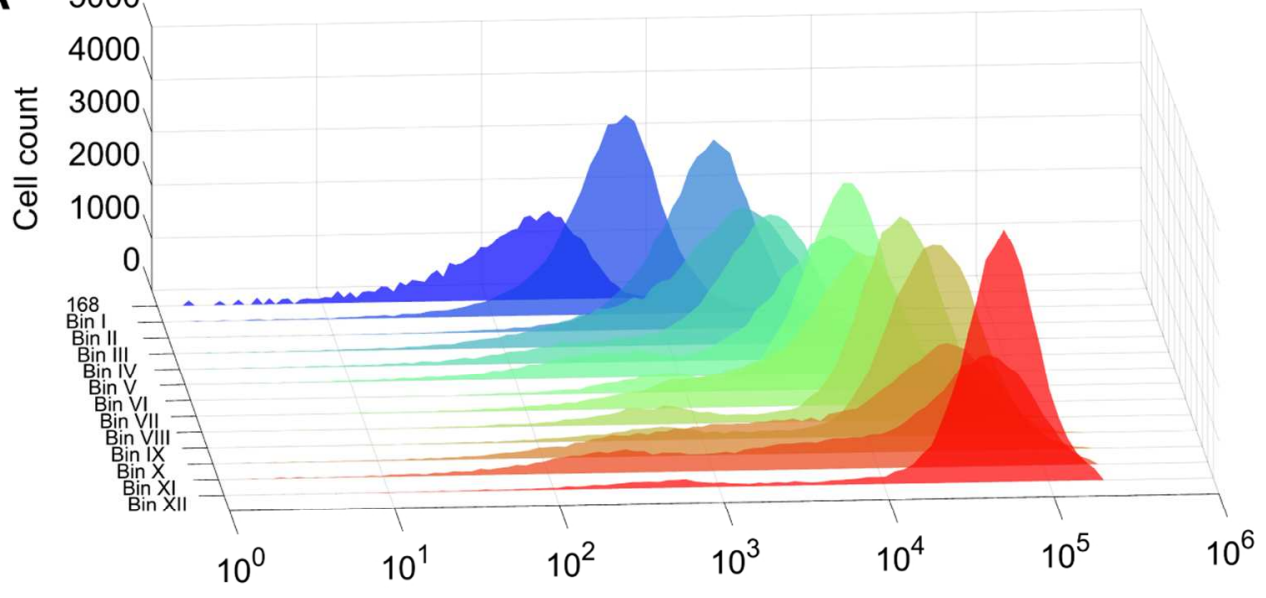

B 6000

GFP fluorescence $(A U)$

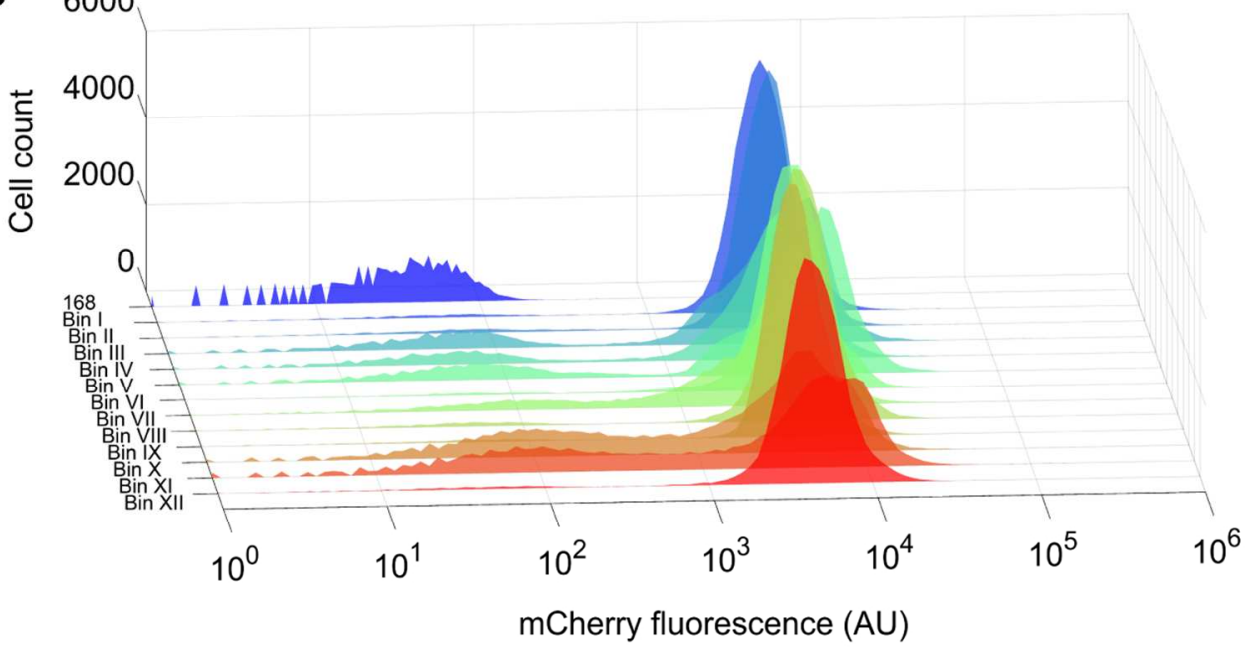

Figure 3: GFP and mCherry fluorescence of bins I to XII. The populations of the 12 bins and the parent strain 168 were cultured and 50,000 events of each population were analyzed by FACScan. (A) The cell counts of GFP fluorescence and (B) mCherry fluorescence intensities are shown.

$111 \times 115 \mathrm{~mm}(300 \times 300 \mathrm{DPI})$ 

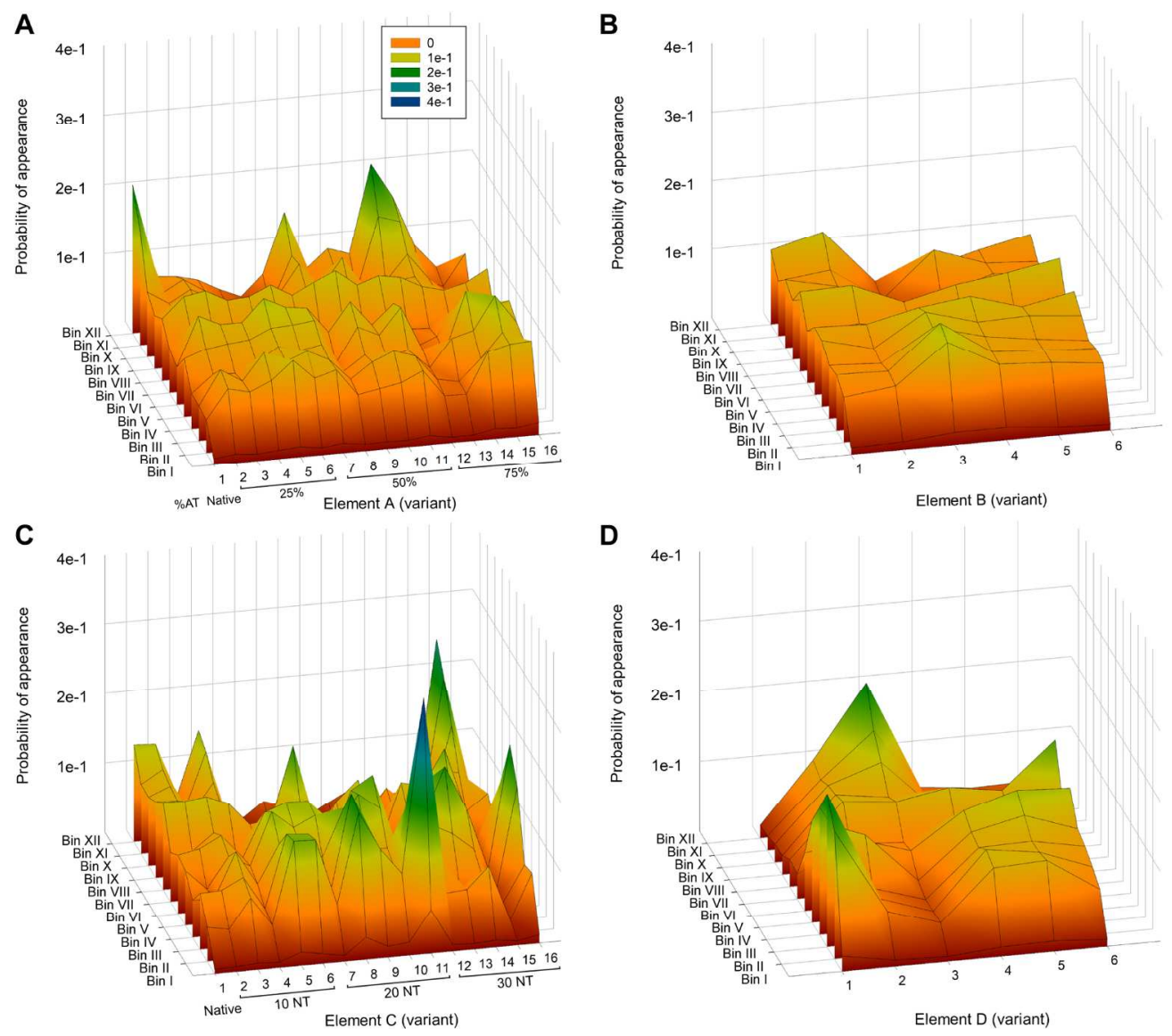

Figure 4: Abundance of SEM Elements in bins I to XII. The abundance of Element A, Element B, Element C and Element $D$ variants over the twelve bins were quantified and normalized against their total counts in the SEM library, respectively. Bin I represents the lowest GFP fluorescence and bin XII the highest GFP fluorescence. The frequencies of (A) 16 Element A variants with different \%AT content, (B) 6 Element B variants, (C) 16 Element $C$ variants with different spacer length and (D) 6 Element $D$ variants are shown. Variant 1 represents the native veg expression module. The probabilities of appearance are color coded and shown in panel $A$.

$177 \times 155 \mathrm{~mm}(300 \times 300 \mathrm{DPI})$ 
Figure 5: Heat map of all element variants distributed over the 12 bins. Hierarchical clustering of the elements using correlation as distance metric and complete linkage as agglomeration method. Bins are normalized by Z-scores. High relative abundance is white and low relative abundance is red.

\section{$84 \times 131 \mathrm{~mm}(300 \times 300$ DPI $)$}



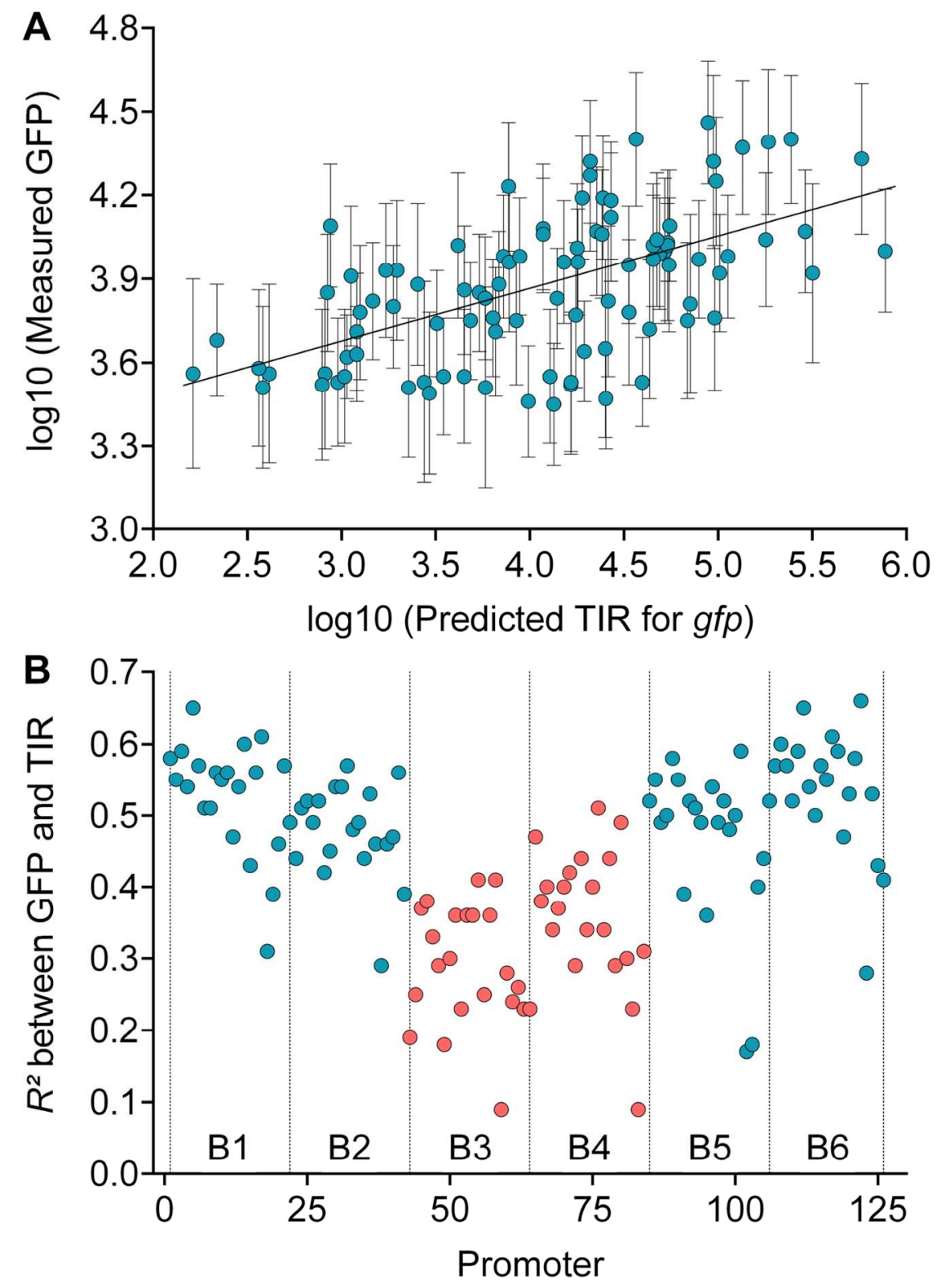

Figure 6: Measured GFP levels and predicted translation initiation. (A) The log10 of the average GFP expression level of the 96 RBS modules (sixteen C elements times six D elements $=96$ ) including their standard deviation was plotted against the log10 of the predicted translation initiation rate (TIR) calculated by the RBS calculator V2.0 26 and resulted in a moderate positive correlation $(R 2=0.56)$. (B) Correlating GFP expression and predicted translation initiation rate (TIR) for each of the 126 promoter sequences on the $\mathrm{x}$-axes (twenty-one A elements times six B elements $=126$ ). First 32 promoters with Elements B1 and B2 are colored blue, promoters 33-64 with Elements B3 and B4 are colored red and promoters 65-96 with Elements B5 and B6 are colored blue.

$116 \times 160 \mathrm{~mm}(300 \times 300 \mathrm{DPI})$ 

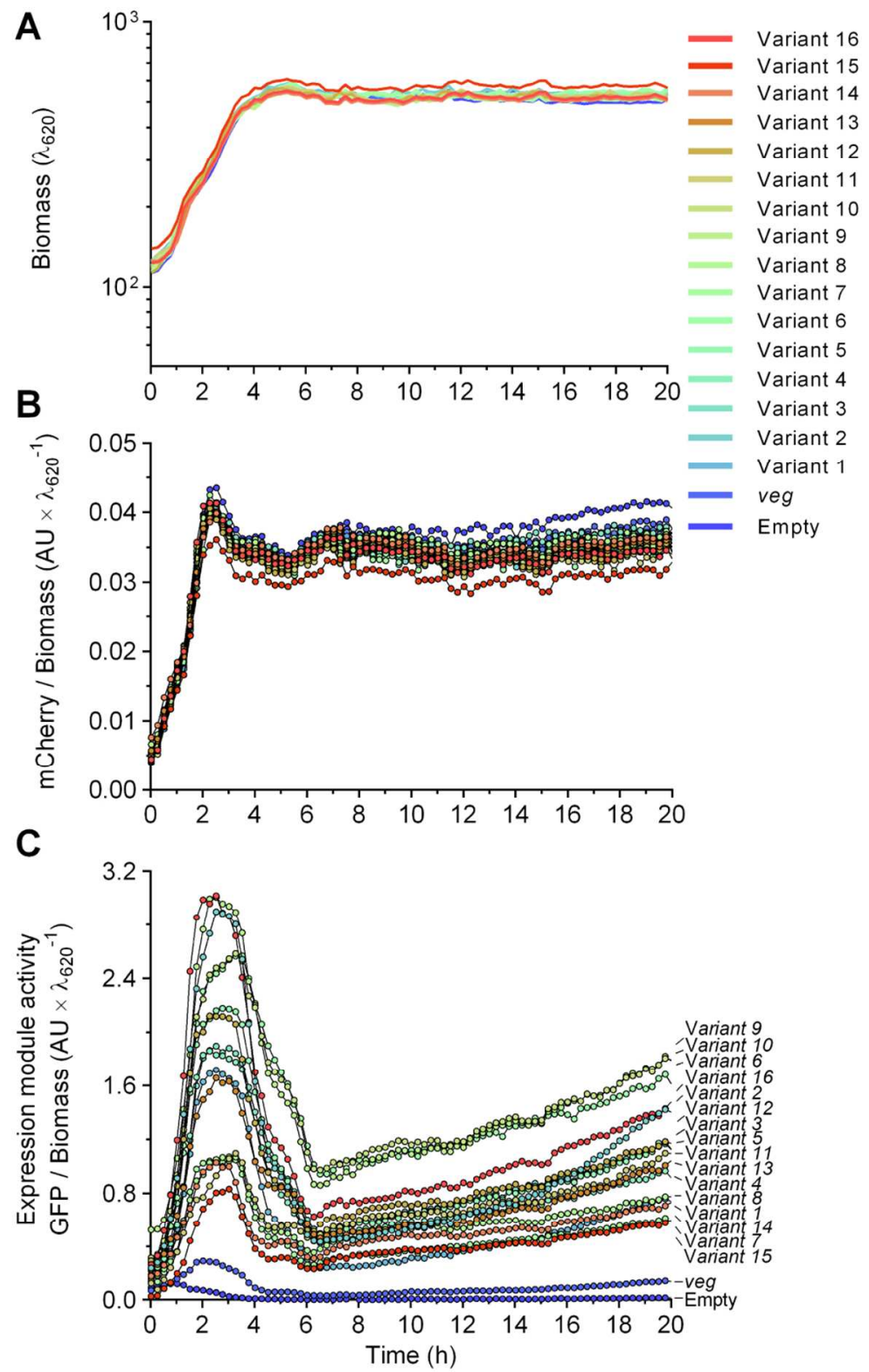

Figure 7: Growth, mCherry and GFP over time. Strains containing sixteen selected SEMs and control strains carrying no expression unit (empty) or native expression module (veg) were analyzed during growth. (A) Biomass increase, (B) production of mCherry over biomass and (C) SEM activity reflected by GFP

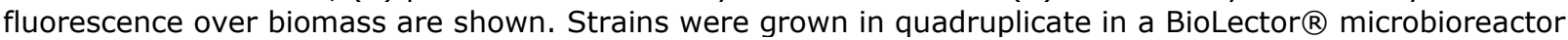
for $20 \mathrm{~h}$ in LB medium and the average values are shown.

$84 \times 135 \mathrm{~mm}(300 \times 300$ DPI $)$ 

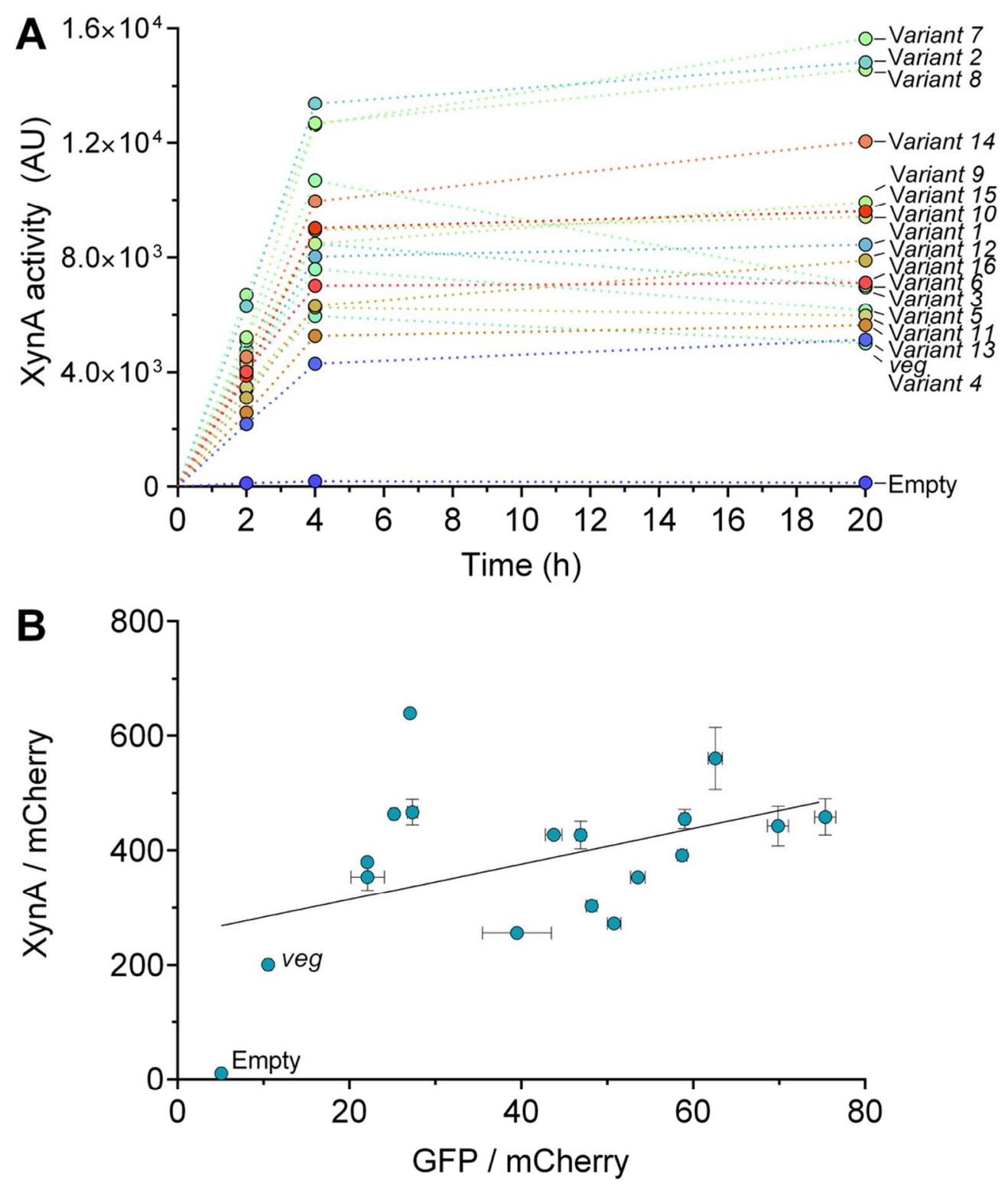

Figure 8: Xylanase production of selected SEMs and analysis of interaction between GFP and XynA expression. Strains containing 16 expression modules and control strains carrying no expression module (empty) or the native expression module (veg) were analyzed for (A) XynA activity in the culture medium during exponential (2 hours), transition (4 hours) and stationary phase (20 hours). (B) Correlation analysis between XynA and GFP production levels with respect to mCherry expression during logarithmic growth phase $(2$ hours) $(R 2=0.20)$. Error bars indicate the standard deviation of four biological replicates.

$99 \times 118 \mathrm{~mm}(300 \times 300 \mathrm{DPI})$ 


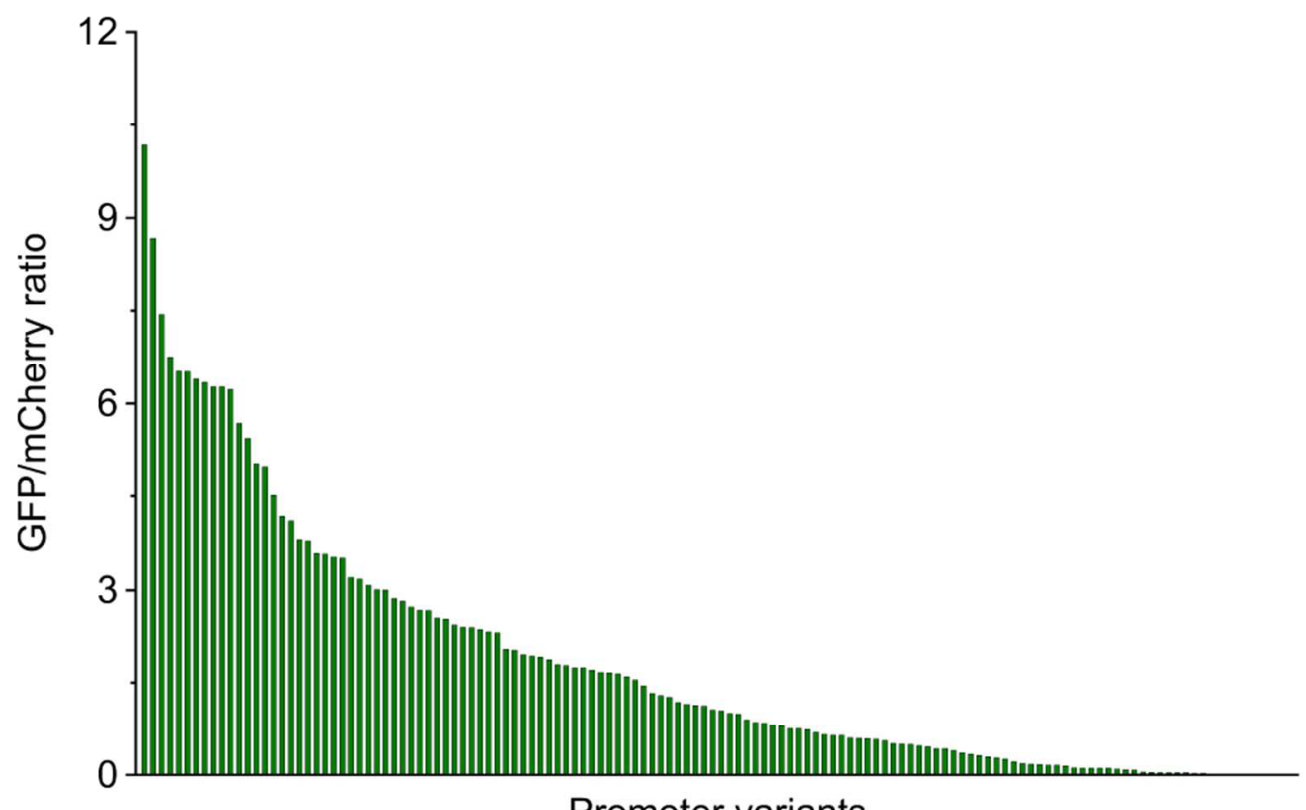

Figure S1: GFP/mCherry ratio of library variants after overnight growth. 135 colonies containing the synthetic expression modules were picked randomly from agar plates and grown overnight. Subsequently, end point measurements of GFP and mCherry were performed in a plate reader to determine expression strength, as reflected by the GFP/mCherry ratio.

\section{$84 \times 54 \mathrm{~mm}(300 \times 300$ DPI $)$}




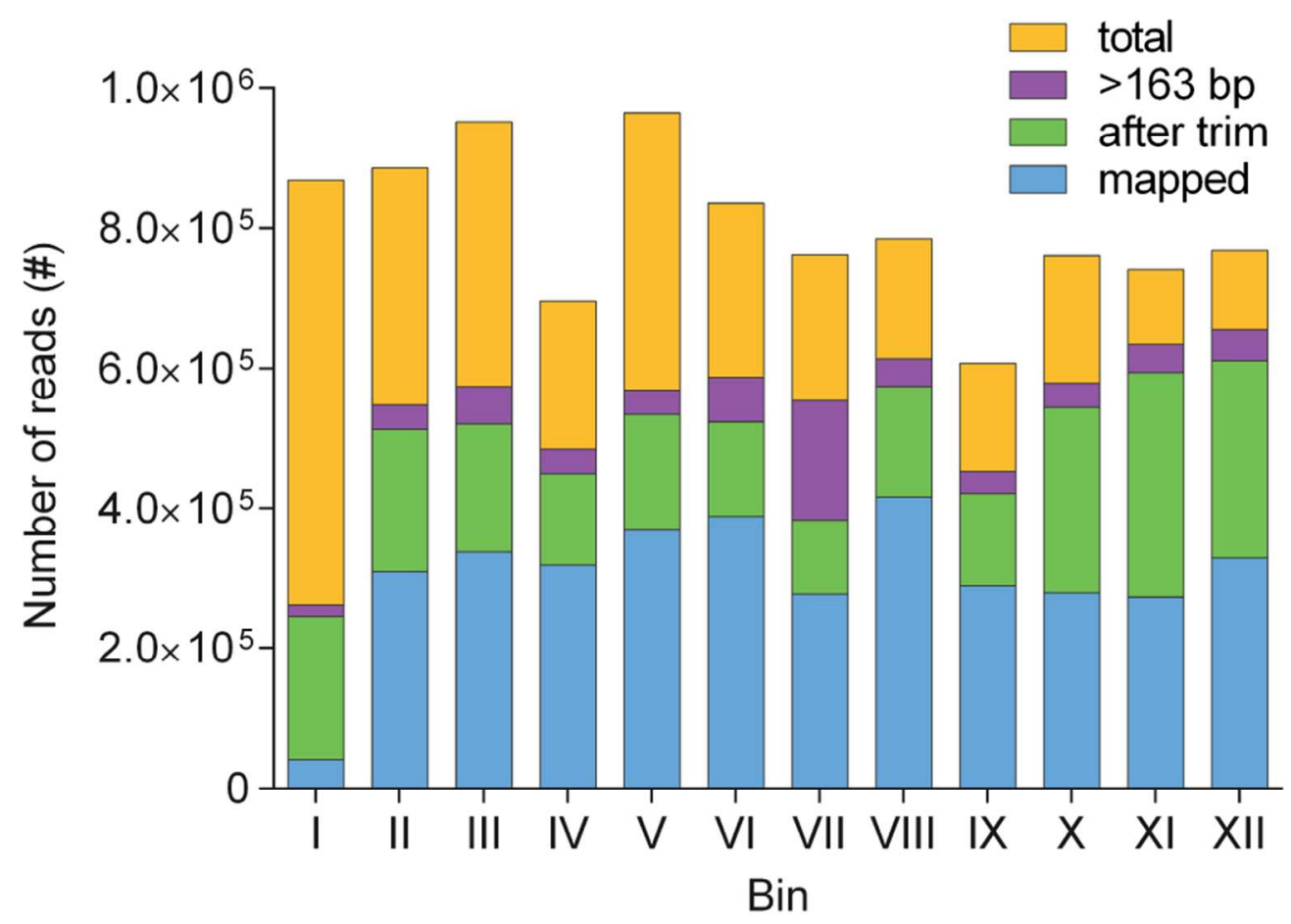

Figure S2: Illumina HiSeq 2500 sequence statistics of twelve bins containing SEMs. The yellow bars represent the total number of reads, followed by the length filtering $>163$ bp (purple) and the filtering for after trimming $5^{\prime}$ amyE and gfp sequences (green). The cyan bars represent the total number of reads that were mapped to the design library. $91.37 \%$ of the 12,000 designed SEMs were identified in the sequence data.

$84 \times 60 \mathrm{~mm}(300 \times 300 \mathrm{DPI})$ 


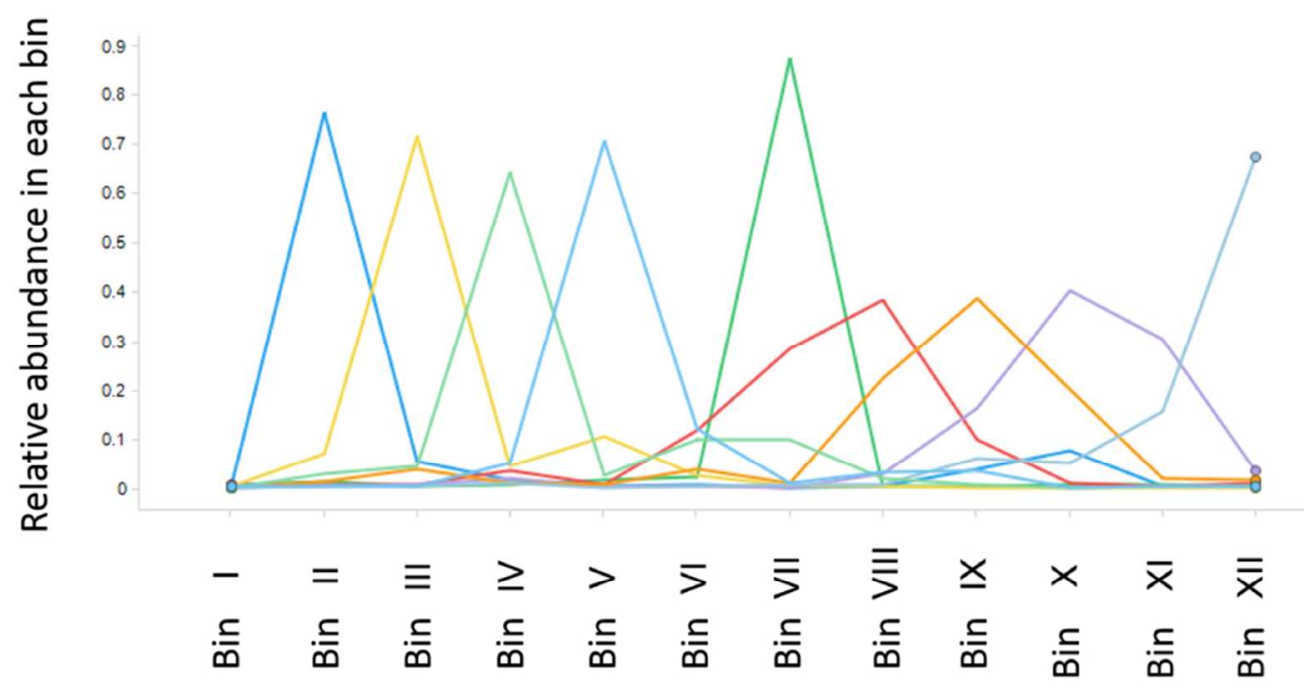

Figure S3: Relative abundance of nine arbitrary SEMs over the twelve bins. The differential sequence distribution of nine arbitrary sequences over all bins is shown.

$84 \times 46 \mathrm{~mm}(300 \times 300$ DPI $)$ 

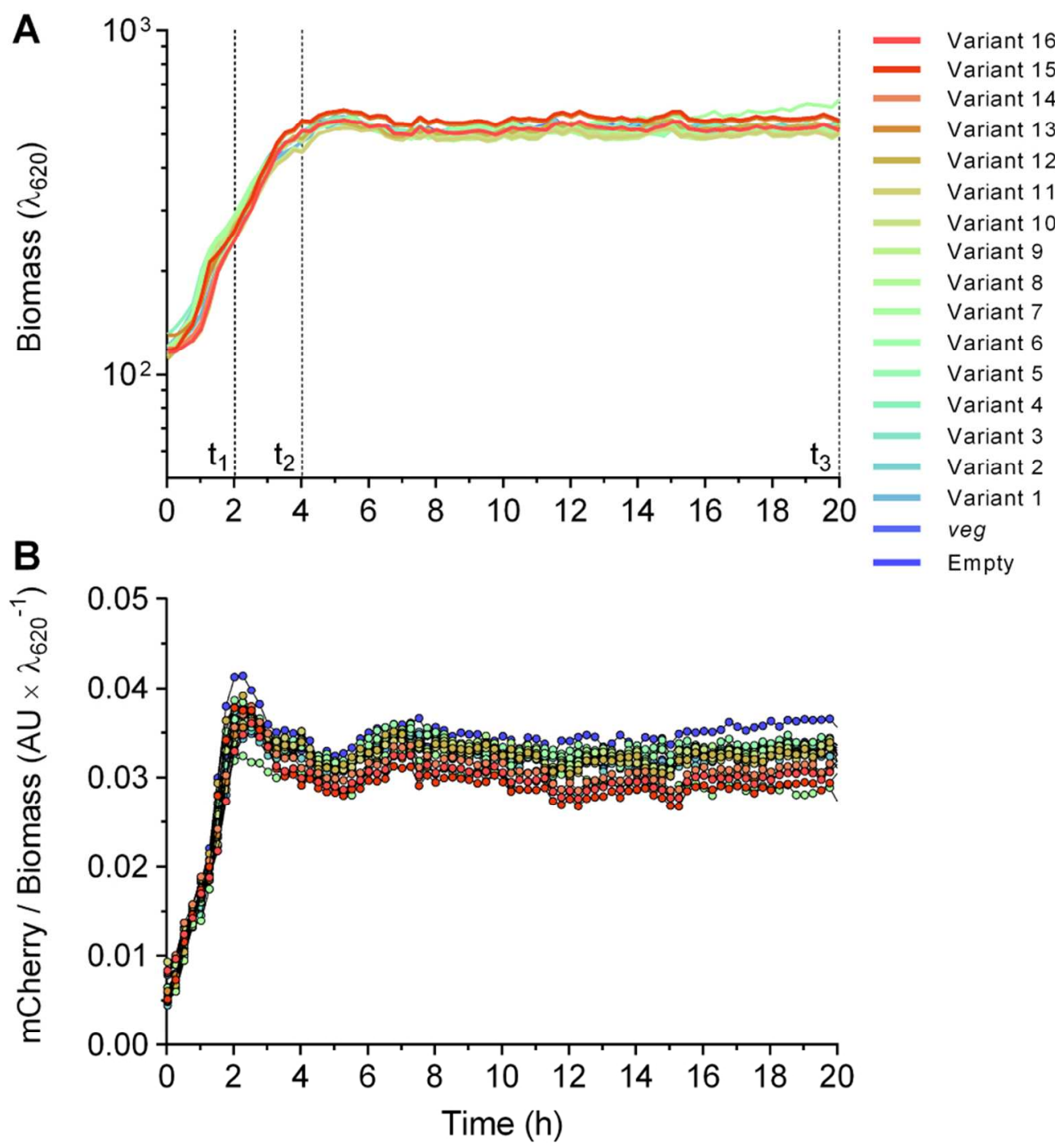

Figure S4: Growth and mCherry production of strains expressing XynA over time. Strains containing sixteen selected SEMs, no expression module (empty) or the native expression module (veg) were analyzed during growth. (A) Biomass increase and (B) production of mCherry per unit biomass are shown. Strains were grown in quadruplicate in a BioLector ${ }^{\circledR}$ for 20 hours in LB medium and the average values are shown.

$84 \times 90 \mathrm{~mm}(300 \times 300 \mathrm{DPI})$ 